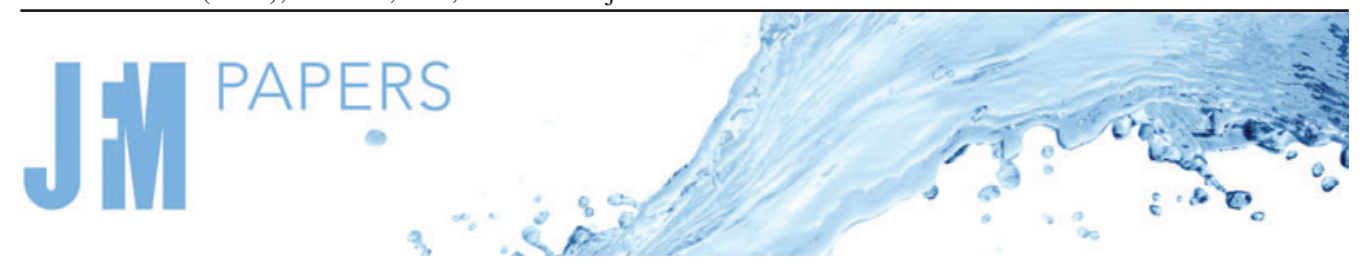

\title{
Turbulent flow in curved channels
}

\section{Geert Brethouwer $\dagger$}

FLOW and SeRC, Department of Engineering Mechanics, KTH Royal Institute of Technology, SE-100 44 Stockholm, Sweden

(Received 1 July 2021; revised 21 September 2021; accepted 25 October 2021)

Fully developed turbulent flow in channels with mild to strong longitudinal curvature is studied by direct numerical simulations. The Reynolds based on the bulk mean velocity and channel half-width $\delta$ is fixed at 20000 , resulting in a friction Reynolds number of approximately 1000 . Four cases are considered with curvature varying from $\gamma=2 \delta / r_{c}=0.033$ to 0.333 , where $r_{c}$ is the curvature radius at the channel centre. Substantial differences between the mean wall shear stress on the convex and concave walls are already observed for $\gamma=0.033$. A log-law region is absent and a region with nearly constant mean angular momentum develops in the channel centre for strong curvatures. Spanwise and wall-normal velocity fluctuations are strongly amplified by curvature in the outer region of the concave channel side. Only near the walls, where curvature effects are relatively weak, do the mean velocity and velocity fluctuation profiles approximately collapse when scaled by wall units based on the local friction velocity. Budgets of the streamwise and wall-normal Reynolds-stress equations are presented and turbulence structures are investigated through visualizations and spectra. In the case with strongest curvature, the flow relaminarizes locally near the convex wall. On the concave channel side, large elongated streamwise vortices reminiscent of Taylor-Görtler vortices develop for all curvatures considered. The maximum in the premultiplied two-dimensional wall-normal energy spectrum and co-spectrum shifts towards larger scales with increasing curvature. The large scales substantially contribute to the wall-normal velocity fluctuations and momentum transport on the concave channel side.

Key words: turbulent boundary layers

$†$ Email address for correspondence: geert@mech.kth.se

(C) The Author(s), 2021. Published by Cambridge University Press. This is an Open Access article, distributed under the terms of the Creative Commons Attribution licence (https://creativecommons. org/licenses/by/4.0/), which permits unrestricted re-use, distribution, and reproduction in any medium, provided the original work is properly cited. 


\section{G. Brethouwer}

\section{Introduction}

Turbulent flows with streamline curvature are found in many engineering applications with curved walls. Streamline curvature has a surprisingly large influence on turbulent shear flows and causes a significant change in turbulence intensity and structure even when the extra strain rate induced by streamline curvature is much smaller than the mean shear rate (Bradshaw 1973; Hunt \& Joubert 1979), implying that streamline curvature directly affects the turbulence.

Effects of wall curvature on turbulent boundary layers has been studied quite extensively experimentally. Muck, Hoffmann \& Bradshaw (1985) measured reduced skin friction and Reynolds stresses in a turbulent boundary layer on a convex wall with a mild curvature of $r_{c} / \delta \approx 100$, where $r_{c}$ is the curvature radius and $\delta$ the boundary layer thickness, and found that the flow rapidly responds when curvature is imposed. Gillis \& Johnston (1983) accordingly found that turbulent length scales were immediately reduced after the onset of strong convex wall curvature with $r_{c} / \delta \approx 10$.

Whereas convex wall curvature acts to stabilize, concave curvature acts to destabilize. Hoffmann, Muck \& Bradshaw (1985) observed significant changes in turbulence structure and distinct longitudinal large-scale vortices in a boundary layer on a mildly concave wall with $r_{c} / \delta \approx 100$. These longitudinal vortices are reminiscent of Taylor-Görtler (TG) vortices produced by centrifugal instability in a concave laminar boundary layer. Barlow \& Johnston (1988) studied the effect of strong concave wall curvature with $r_{c} / \delta \approx 15$ on a turbulent boundary layer, and observed significantly increased skin friction and turbulence intensities, especially of wall-normal fluctuations, compared with a flat plate boundary layer. The concave curvature also led to a growth of the large turbulent scales, but Barlow \& Johnston (1988) did not observe distinct longitudinal vortices; the large scales were unsteady and lacked streamwise coherency. They suggested that spanwise inhomogeneity in the inflow caused by e.g. vortex generators triggers the formation of coherent longitudinal TG-like vortices. The absence of distinct longitudinal vortices in their experiment were likely a result of a more homogeneous inflow. Large-eddy simulations by Lund \& Moin (1996) accordingly showed that an inflow with coherent structures leads to stronger and more distinct TG vortices and higher Reynolds stresses in a concave wall boundary layer.

Fully developed turbulent flow in strongly curved and wide ducts with $r_{c} / \delta=9$ to 19 , where from now on $r_{c}$ is the curvature radius at the channel centreline and $\delta$ the channel half-gap width, was experimentally studied by Wattendorf (1935) and Eskinazi \& Yeh (1956). In the channel core region $r U$ was approximately constant and therefore the mean flow nearly irrotational. Here, $U$ is the mean streamwise velocity and $r$ the curvature radius. Wattendorf (1935) observed only a slight increase in flow resistance as a result of curvature, although Eskinazi \& Yeh (1956) found that the wall shear stress is much larger and turbulence stronger on the outer channel side, in accordance with concave and convex wall effects, respectively, on turbulent boundary layers. Hunt \& Joubert (1979) experimentally investigated the effect of weak curvature with $r_{c} / \delta=200$ on turbulent flow in a curved channel with a length of $150 \delta$. No irrotational mean flow was observed but instead a noticeable difference in the shear stress on the inner and outer walls of approximately $20 \%$, a secondary flow in the centre region caused by TG vortices and change in turbulence anisotropy.

Direct numerical simulation (DNS) of fully developed turbulent flow in a mildly curved channel with $r_{c} / \delta=79$ at a friction Reynolds number $R e_{\tau}=u_{\tau} \delta / \nu=168$ where $u_{\tau}$ is the friction velocity and $v$ the viscosity was carried out by Moser \& Moin (1987). The computational domain was $12.64 \delta$ long and $4 \pi / 3 \delta$ wide and could accommodate one pair 


\section{Turbulent flow in curved channels}

of counter-rotating TG vortices. The TG vortices contributed noticeably to the turbulent shear stress on the concave side, but no drastic growth in wall-normal velocity fluctuations was observed. Nagata \& Kasagi (2004) performed DNSs of mildly to strongly curved channel flows with $r_{c} / \delta=77$ to 5 at $R e_{\tau}=150$ with a domain $2.5 \pi \delta$ long and $\pi \delta$ wide. A strong growth of wall-normal velocity fluctuations and an approximately irrotational mean flow in the centre were observed but they claimed rather surprisingly that the TG vortices contributed to turbulence production on the convex and not the concave side. Matsubara \& Miura (2017) and Matsubara \& Muromoto (2019) studied a developing flow in a strongly curved channel with planar inflow with $R e_{\tau}$ up to 550 by DNS. Large-scale structures grow and intensify going downstream according to visualizations, spectra and two-point correlations. Patel \& Sotiropoulos (1997) further reviewed earlier studies of streamline curvature effects on turbulent shear flows.

Concave and convex streamline curvature have similar (de)stabilizing effects on turbulent wall flows as anticyclonic and cyclonic rotation about the spanwise axis, respectively, as noted by Bradshaw (1969) and Hunt \& Joubert (1979). Moreover, the irrotational mean flow observed in strongly curved channels (Wattendorf 1935) is analogous to the absolute-zero-mean-vorticity region observed in rotating channel flow (Brethouwer 2017) as both correspond to neutral stability (Brauckmann, Salewski \& Eckhardt 2016), and anticyclonic rotation and concave streamline curvature both cause an energy transfer from the streamwise to wall-normal Reynolds stresses (Hunt \& Joubert 1979; Brethouwer 2017). Since system rotation and streamline curvature have a significant impact on turbulent flows in many engineering applications, it is important to incorporate their effects in turbulence models. Durbin (2018) reviews methods to include rotation and curvature effects in a unified way in turbulence models. However, their effects on Reynolds stresses and turbulent structures are not fully equivalent and the extent of the similarities between turbulent wall flows subject to streamline curvature and rotation has to be assessed yet.

Although streamline curvature effects on shear flows have been studied quite a lot, we nevertheless have a limited understanding of how the flow, turbulence and TG vortices change with curvature since previous studies were mostly limited to a narrow curvature range or low Reynolds numbers. Here, we present a DNS study of fully developed turbulent flows in mildly to strongly curved channels with a larger computational domain and higher $R e_{\tau} \approx 1000$ than in previous DNS studies. In this geometry there are no ambiguities about inflow conditions, as in some experimental studies, or questions as to whether or not the flow is fully developed, i.e. one-point statistics vary only in the wall-normal direction. However, some interaction between the inner and outer wall regions occurs, which prevents a complete separation of convex and concave streamline curvature effects, as in boundary layers on curved walls. Further, the use of streamwise periodic boundary conditions leads to an inflow with more coherent structures. As such, the TG vortices may be stronger and more coherent than in flows with a more incoherent inflow. The use of quite restricted computational domains in Moser \& Moin (1987) and Nagata \& Kasagi (2004) may have contributed to the formation of unnaturally coherent TG vortices with an enforced spanwise wavelength. Using larger computational domains may alleviate these problems. We study mild to strong streamline curvature effects on the turbulent stresses and large-scale structures at high enough $R e_{\tau}$ to have a separation between turbulence scales related to the near-wall cycle and TG vortices. We also discuss similarities between curved and spanwise rotating channel flows. With this study, we aim to advance our understanding of curvature effects and to aid turbulence modelling efforts. The results of this study can be used to further explore similarities between turbulent flows subject to streamline curvature and rotation. 


\section{G. Brethouwer}

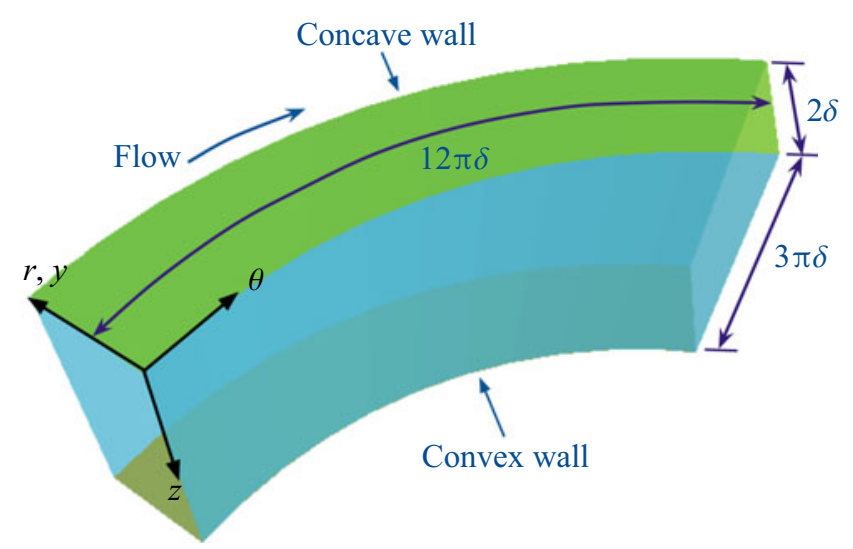

Figure 1. Illustration of the computational domain for curved channel flow. The wall-normal direction $y$ is from the convex (inner) wall towards the concave (outer) wall.

\section{Numerical set-up}

We have carried out four DNSs of fully developed turbulent flows in mildly to strongly curved channels. The incompressible Navier-Stokes equations in cylindrical coordinates are solved in a domain bounded by sectors of two concentric cylinders, as in Moser \& Moin (1987), see figure 1 for an illustration of the computational domain. Streamline curvature effects on turbulent flow in curved channels as in figure 1 are similar to rotation effects on turbulent plane channel flows subject to clockwise rotation about the spanwise axis. Fourier representations with periodic boundary conditions are used in the homogeneous streamwise (azimuthal) and spanwise (axial) directions and a sixth-order compact-finite-difference scheme is used in the wall-normal (radial) direction with no-slip conditions at the walls (Boersma 2011; Peeters et al. 2016). The solution is advanced by a pressure correction method together with a fourth-order Runge-Kutta method for time integration. The Poisson equation for the pressure is solved directly using Fourier transforms in the streamwise and spanwise directions and LU factorization of the matrix resulting from the discrete Poisson equation in the radial direction. The computational domain has a streamwise length of $12 \pi \delta$ along the centreline and a spanwise width of $3 \pi \delta$, which is equally wide but 1.5 times longer than in recent DNS of plane channel flow (Lee \& Moser 2015) to accommodate the long structures, and three times longer and more than two times wider than in the DNS by Moser \& Moin (1987). In the DNS with strongest curvature the domain captures an entire cylinder circumference.

The bulk Reynolds number $R e=U_{b} \delta / v$ is fixed at 20000 by enforcing a constant mass flow rate, giving a $R e_{\tau}=u_{\tau} \delta / \nu$ of approximately 1000 . Here, $U_{b}$ is the bulk mean velocity, $u_{\tau}$ friction velocity defined later and $v$ viscosity. The number of grid points, resolution, curvature and friction Reynolds numbers together with the reference names of the four DNSs are listed in table 1. From now on, curvature refers to $\gamma=2 \delta / r_{c}$, where $r_{c}$ is again the curvature radius at the channel centreline. Run R0 is a DNS of plane channel flow at the same $R e$ with a domain $8 \pi \delta$ long and $3 \pi \delta$ wide, taken from Brethouwer (2017). Here, $R e_{\tau}^{i}$ and $R e_{\tau}^{o}$ are the friction Reynolds numbers based on the friction velocities $u_{\tau i}$ and $u_{\tau o}$ for the inner and outer walls, respectively.

The streamwise and spanwise resolutions in all DNSs are $r \Delta \theta^{+} \leq 13$ and $\Delta z^{+} \leq 6.5$, respectively, in viscous wall units and Fourier modes, comparable to the resolutions used by Lee \& Moser (2015). The resolution is coarsest near the outer wall since, there, the 


$\begin{array}{lcllrcc}\text { Run } & r_{c} / \delta & \gamma & R e_{\tau} & R e_{\tau}^{i} & R e_{\tau}^{o} & N_{x} \times N_{y} \times N_{z} \\ \text { R0 } & \infty & 0 & 1000 & 1000 & 1000 & 2560 \times 385 \times 1920 \\ \text { R1 } & 60 & 0.033 & 1022 & 927 & 1103 & 3840 \times 382 \times 1920 \\ \text { R2 } & 30 & 0.067 & 1030 & 905 & 1126 & 3840 \times 382 \times 1920 \\ \text { R3 } & 12 & 0.167 & 1030 & 852 & 1135 & 3840 \times 382 \times 1920 \\ \text { R4 } & 6 & 0.333 & 1020 & 764 & 1109 & 4096 \times 382 \times 1920\end{array}$

Table 1. Run name, channel curvature, friction Reynolds numbers and number of streamwise, wall-normal and spanwise grid points $N_{x}, N_{y}, N_{z}$, respectively.

friction velocity is higher and the domain longer. Grid points in the radial direction are clustered near the wall with at least 7 grid points in the viscous sublayer $y^{+} \leq 5$ and the first grid point at $y^{+}<0$.5. Some validations of the numerical methodology are presented in Appendix A.

The balance between mean streamwise pressure gradient $\mathrm{d} P /\left(r_{c} \mathrm{~d} \theta\right)$, the total mean wall shear stress $\tau_{\text {tot }}$ and friction velocities is given by

$$
u_{\tau}^{2}=\frac{\tau_{t o t}}{\rho}=-\frac{\delta}{\rho r_{c}} \frac{\mathrm{d} P}{\mathrm{~d} \theta}=\frac{r_{i}^{2} u_{\tau i}^{2}+r_{o}^{2} u_{\tau o}^{2}}{2 r_{c}^{2}}
$$

where $r_{i}$ and $r_{o}$ is the inner and outer wall radius, respectively, $P$ the mean pressure and $\rho$ the density. The total mean shear stress obeys

$$
\bar{\tau}=v\left(\frac{\partial U}{\partial r}-\frac{U}{r}\right)-\overline{u v}=\frac{1}{4 r^{2} r_{c} \delta}\left[r_{i}^{2}\left(r_{o}^{2}-r^{2}\right) u_{\tau i}^{2}+r_{o}^{2}\left(r_{i}^{2}-r^{2}\right) u_{\tau o}^{2}\right],
$$

where $U$ is the mean streamwise velocity, $u$ and $v$ streamwise and wall-normal velocity fluctuations and an overline implies averaging in time and homogeneous azimuthal and axial directions. The derivation is given in Appendix B.

The DNSs are run for a sufficiently long time to reach a statistically stationary state and then run for at least $280 \delta / U_{b}$ to obtain well-converged statistics.

\section{Flow statistics}

First, mean flow, wall shear stress and turbulent fluctuation statistics are presented. In the next sections, Reynolds-stress budgets and flow structures are studied. In the following figures $y=\left(r-r_{c}\right) / \delta$, with $y=-1$ and 1 at the inner (convex) and outer (concave) wall, respectively. Many figures include results of plane channel flow case R0. Similarities between the present curved channel flow cases and plane channel flow rotating about the spanwise axis are expected, as noted in the Introduction, and also further discussed. The mean streamline turning rate is $U / r$ in curved channel flow, with $U$ the mean streamwise velocity. This suggests replacing the rotation rate $\Omega$ in the rotation number $R o=2 \Omega \delta / U_{b}$ of spanwise rotating channel flow by $U_{b} / r_{c}$ to obtain that an equivalent rotation number for curved channel flow of $R o=2 \delta / r_{c}=\gamma$. Thus, it appears meaningful to compare rotating and curved channel flows for $R o=\gamma$ when rotation and curvature effects are comparable. In Brethouwer (2017), results of spanwise rotating channel flow DNS at $R e=20000$ are reported, but only one case with $R o=0.15$ has a $R o$ that falls into the range of curvatures $\gamma \simeq R o \leq 0.333$ considered here. The other cases have $R o \geq 0.45$, corresponding to much stronger curvatures. Results of the rotating channel flow DNS 


\section{G. Brethouwer}

at $R e=20000$ and $R o=0.15$ are included in some figures to make a few comparisons possible.

Figure 2(a) shows mean streamwise velocity profiles $U / U_{b}$ in the curved channel flow DNSs. The mean shear is remarkably weak for $0 \lesssim y \lesssim 0.6$ and the velocity gradient is even slightly positive at the centreline in the mild curvature cases R1 and R2 but the profiles become more oblique for increasing curvature. This is owing to the development of a core region in runs R1 to R4 with a nearly constant mean specific angular momentum $r U$, see figure $2(b)$, as observed in previous experiments of strongly curved duct flows with $r_{c} / \delta \leq 19$ (Wattendorf 1935; Eskinazi \& Yeh 1956). Here, we see that an irrotational mean flow region tends to emerge for $r_{c} / \delta \leq 30$, skewed towards the outer concave side and expanding with increasing curvature. Fluid elements thus tend to preserve angular momentum when being displaced by eddies in the radial direction. A core region with nearly constant angular momentum also develops in turbulent Taylor-Couette flows and implies a trend towards neutral stability according to Rayleigh's inviscid criterion for centrifugal instability (Brauckmann et al. 2016). A similar phenomenon occurs in spanwise rotating plane Couette and channel flow, in which a neutrally stable zero-absolute-mean-vorticity region emerges (Brauckmann et al. 2016; Brethouwer 2017). Constant angular momentum furthermore implies that the total production of streamwise Reynolds stress $\overline{u u}$ given by $P_{u u}=-2 \overline{u v}(r \partial(U / r) / \partial r+2 U / r)$ (Hunt \& Joubert 1979) becomes zero. Here, the first term on the right-hand side is the shear production term and the second term an additional production term owing the curvature strain. This additional production term $4 \overline{u v} U / r$ is present as well in the wall-normal Reynolds-stress $\overline{v v}$ transport equation, although with an opposite sign. It can be seen as a redistribution term between the normal Reynolds stresses since it influences turbulence anisotropy, as we see later, but it is absent in the turbulent kinetic energy transport equation (Hunt \& Joubert 1979). In the mild curvature cases a small extra production term due to curvature is still able to offset the shear production term in $P_{u и}$ because the mean shear is very weak, as shown in figure 2(a). The shear production and additional production owing to curvature are studied in the next section. Again, similarities with spanwise rotating shear flows show up: the Coriolis term redistributes energy between the normal Reynolds stresses but does not directly contribute to turbulent kinetic energy. Furthermore, the sum of shear production and Coriolis term in the $\overline{u u}$ transport equation becomes zero in the zero-absolute-mean-vorticity state (Brethouwer 2017).

Figure $2(c, d)$ shows the mean streamwise velocity $U^{*}$ on the inner and outer channel side, respectively, as functions of wall distance $y^{*}$ in local scaling. Local scaling, denoted by a superscript $*$, implies scaling based on the local friction velocity $u_{\tau i}$ and $u_{\tau o}$ at the inner and outer channel wall, respectively. The curved channel profiles away from the wall lie above and below the plane channel flow profile on the convex and concave sides as a result of the reduced and enhanced turbulent shear stresses, respectively (Moser \& Moin 1987), and depart earlier from the R0 profile for stronger curvature. On the concave side all profiles approximately collapse for $y^{*} \lesssim 50$ but on the convex side the R3 and $\mathrm{R} 4$ profiles diverge at smaller $y^{*}$, indicating that strong curvature affects the flow even near the walls. An identifiable log layer is thus absent in these mildly to strongly curved channels. At a given $y^{+}$in the log layer the curvature related production to shear production ratio $(U / r) /(\partial U / \partial r) \sim \delta /\left(\operatorname{Re}_{\tau} r_{c}\right)$. The log layer therefore likely reappears at higher $\operatorname{Re}_{\tau}$ when the boundary layer becomes thinner and shear production grows relative to curvature related production near the wall. Hunt \& Joubert (1979) observed a log-layer profile on both sides in experiments with $R e_{\tau} \approx 700$ to 2700 , but their channel was only weakly curved with $r_{c} / \delta=200$. 
Turbulent flow in curved channels
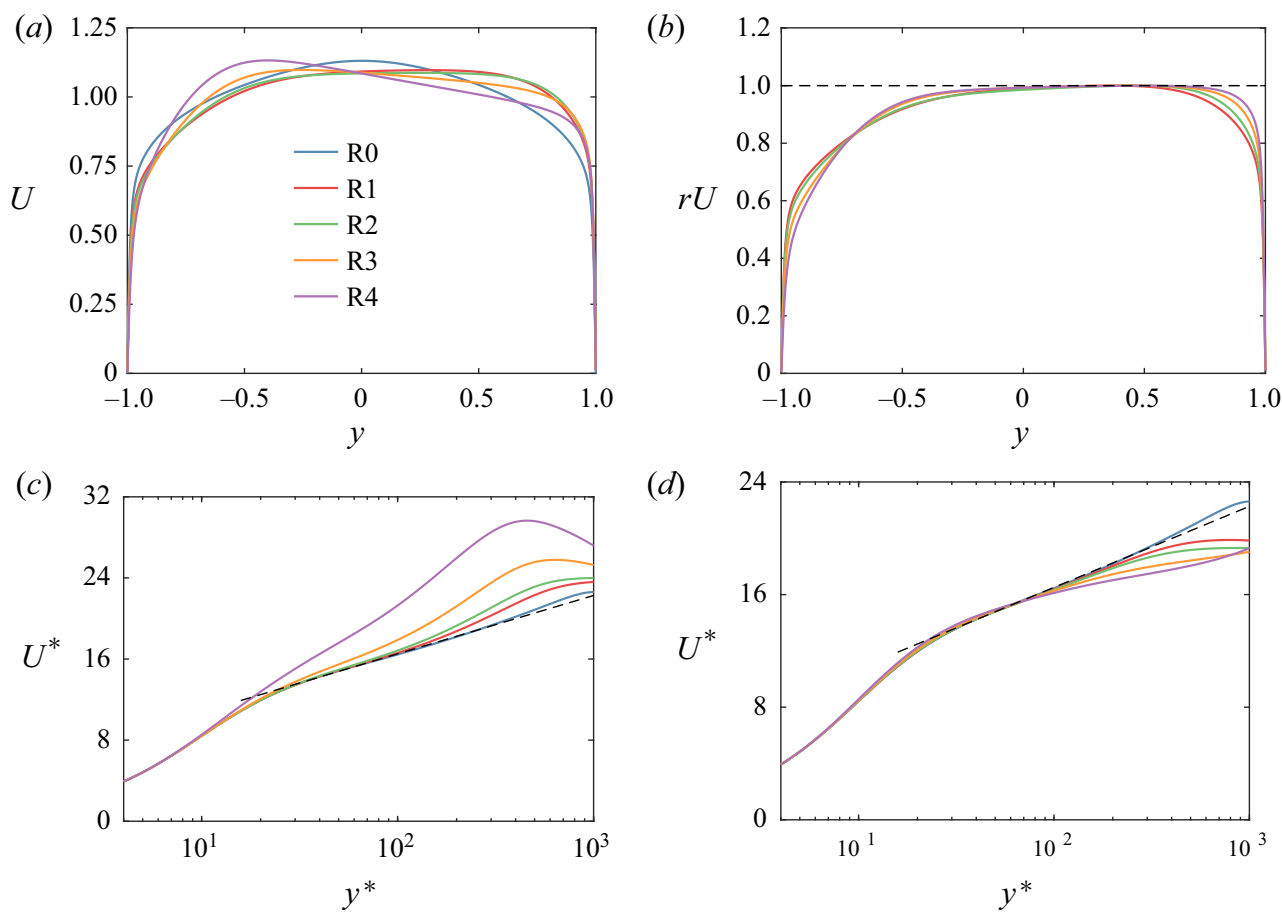

Figure 2. Profiles of the $(a)$ normalized mean streamwise velocity $U / U_{b}$ and $(b)$ specific angular momentum $r U$ scaled by its maximum value. Panels $(c)$ and $(d)$ show $U^{*}$ as functions of $y^{*}$ on the convex (inner) and concave (outer) channel side, respectively.

Figure 3(a) shows computed total mean shear stress $\bar{\tau}$ profiles, i.e. the sum of viscous and turbulent shear stresses, scaled by $\tau_{\text {tot }}$ defined in (2.1). The profiles show the expected growth and decline of shear stress magnitude on the convex and concave side, respectively, and collapse on the derived profile given by (2.2), implying that the statistics are well converged. The mean wall shear stresses $\tau_{w}$ on the inner and outer walls, shown in figure 3(b), deviate significantly from that of the plane channel flow case R0 already for the weakest curvature considered. For curvatures larger than in case R2, $\tau_{w}$ does not grow much more and actually starts to decline on the concave side whereas on the convex side $\tau_{w}$ declines monotonically with curvature owing to a continuing weakening of turbulence. The total mean wall shear stress $\tau_{\text {tot }}$ and related flow resistance in the present curved channel flows, on the other hand, are only moderately higher than in plane channel flow, consistent with observations by Wattendorf (1935). Data of spanwise rotating channel flow DNS at $R e=20000$ and $R o=0.15$ (Brethouwer 2017) are also included in figure 3(b). Figure 3(b) indicates that, at least for moderate $R o$, the effects of rotation and curvature on mean wall shear stresses are comparable.

Root-mean-square profiles of all velocity fluctuation components and Reynolds shear stresses in global scaling, i.e. scaled by $u_{\tau}$, are shown in figure 4 . In the convex near-wall region the velocity fluctuations monotonically decline with curvature, concurrently with the mean wall shear stress, whereas the near-wall peak of the streamwise and wall-normal fluctuations does not vary much with curvature on the concave side. On the other hand, spanwise and especially wall-normal fluctuations drastically grow and streamwise fluctuations decline in the outer concave region for strong curvatures. This growth and decline can be explained by the action of the extra curvature production term, which 


\section{G. Brethouwer}
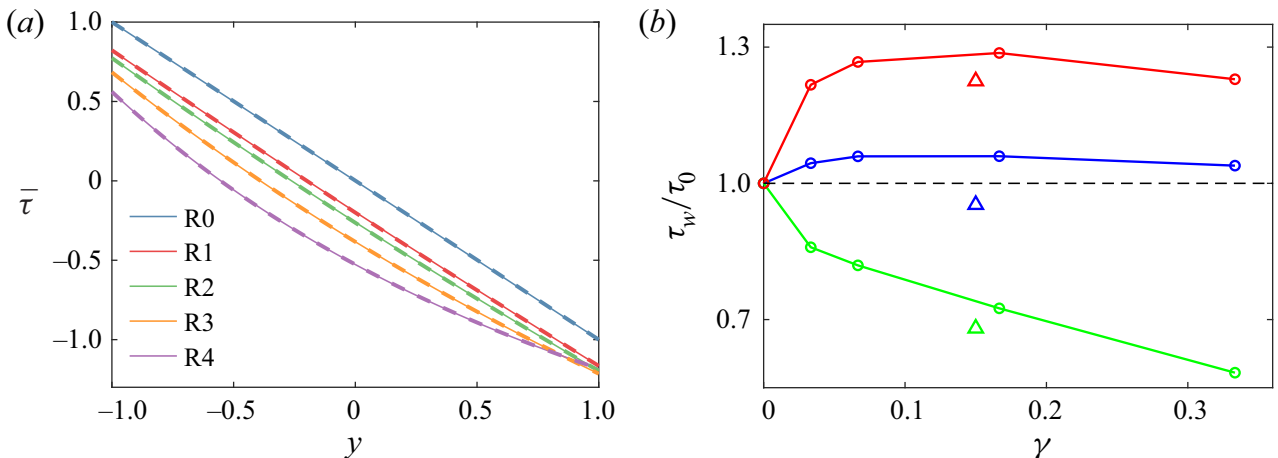

Figure 3. (a) Total mean shear stress $\bar{\tau}$ profiles scaled by $\tau_{\text {tot }}$. Thin solid lines are DNS data and thick dashed lines show $\bar{\tau}$ given by (2.2). (b) Mean wall shear stresses $\tau_{w}$ on the inner (green) and outer walls (red) and $\tau_{\text {tot }}$ (blue) scaled by the mean wall shear stress $\tau_{0}$ in plane channel flow (run R0). Each dot represents a DNS. The triangles are corresponding rotating channel flow data at the same $R e$ and $R o=0.15$.
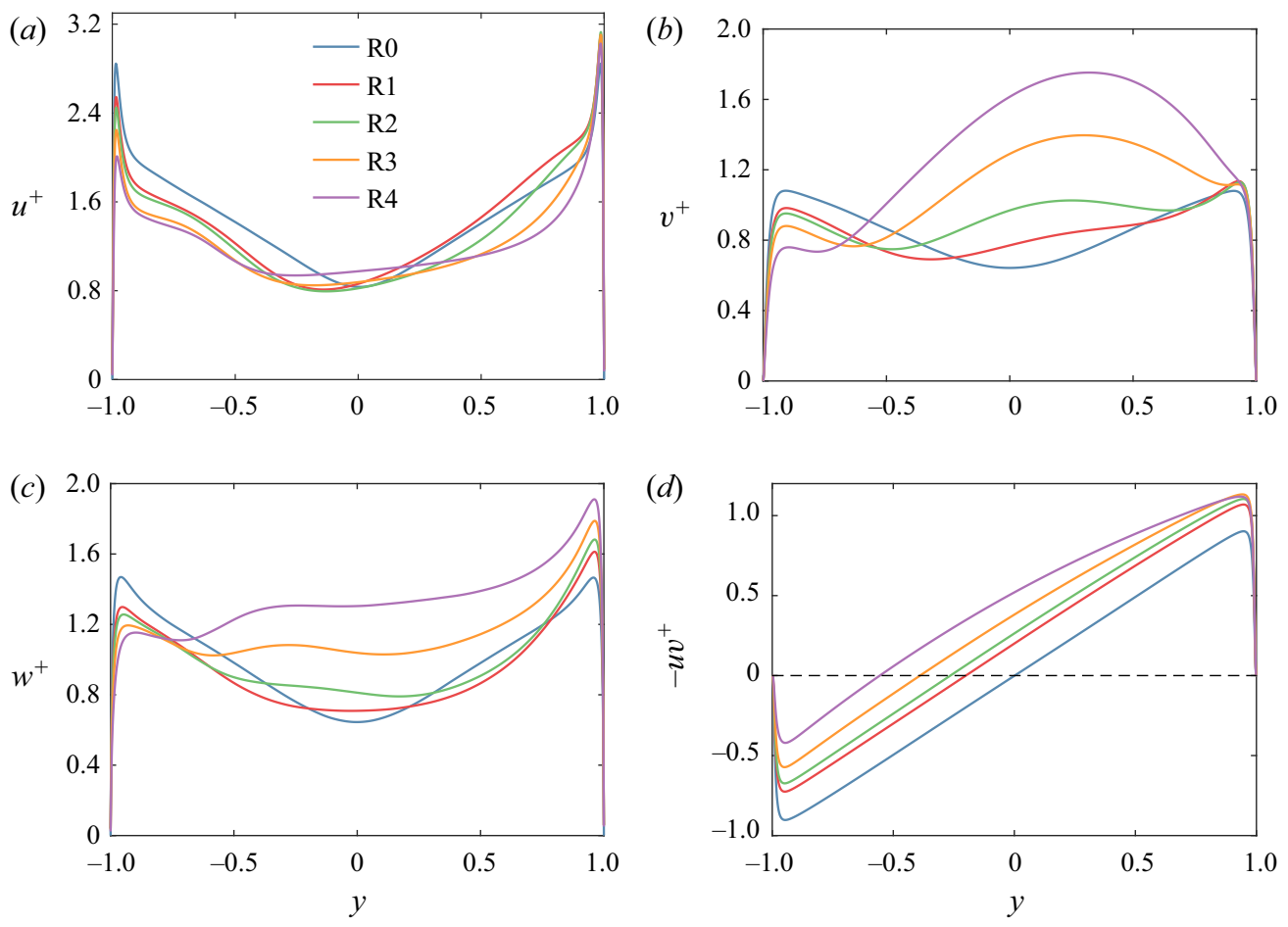

Figure 4. Root-mean-square profiles of $(a)$ streamwise, $(b)$ wall-normal and $(c)$ spanwise velocity fluctuations and $(d)$ profiles of the mean turbulent shear stress $\overline{u v}$ in global scaling.

transfers energy from streamwise to wall-normal fluctuations, as discussed before. Energy transfer between the velocity components due to the extra curvature production is studied in the next section and shown to be relatively small in the near-wall region but significant in the outer region. The growth of wall-normal and spanwise fluctuations comes along with a structural change of turbulence and amplification of large-scale structures on the concave side, as shown later. 
(a)

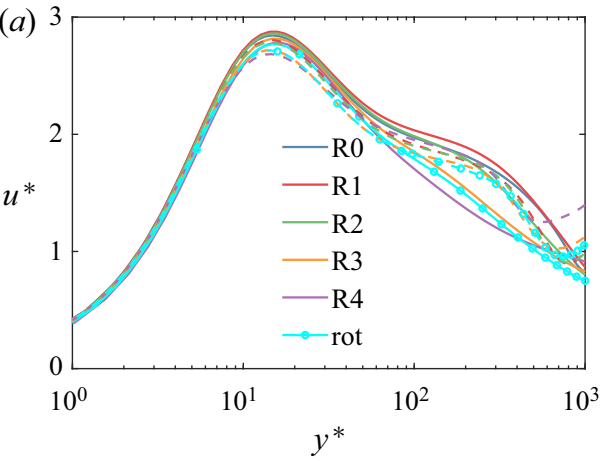

(b)

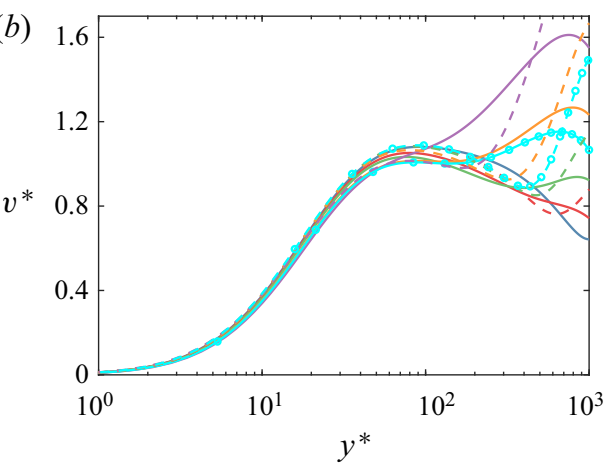

(c)

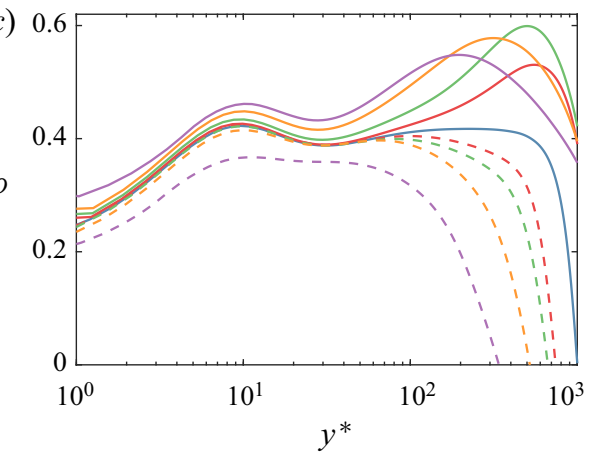

Figure 5. Root-mean-square profiles of the $(a)$ streamwise and $(b)$ wall-normal velocity fluctuations and $(c)$ correlation coefficient $\rho$ on the convex (dashed lines) and concave sides (solid lines) in local scaling. Included in $(a, b)$ are profiles in local scaling (labelled as 'rot') from DNS of spanwise rotating channel flow at $R e=$ 20000 and $R o=0.15$ (Brethouwer 2017) on the cyclonic (dashed line) and anticyclonic sides (solid line).

In local scaling, by contrast, streamwise and wall-normal velocity fluctuations are very similar in the convex and concave near-wall region for different curvatures, see figure $5(a, b)$, suggesting that the near-wall dynamics is mostly unaffected by wall curvature. On the convex side the similarity to plane channel flow extends quite far, up to $y^{*} \approx 200$. On the concave side the similarity ends earlier because profiles of the strongly curved cases diverge beyond the buffer layer. In run R4 the near-wall peak of the wall-normal component disappears as a result of the strong growth of the fluctuations in the outer region caused by a fundamental change in the outer layer dynamics. Near-wall similarity, also observed in experiments of a turbulent boundary layer on a concave wall by Barlow \& Johnston (1988) and in DNS by Moser \& Moin (1987), is to be expected since the strain-rate ratio $(U / r) /(\mathrm{d} U / \mathrm{d} r)$ decreases and thus curvature effects vanish approaching the wall. Curvature effects on the Reynolds stress budgets are accordingly small to moderate in the near-wall region, as shown in the next section, which further supports the expectation that velocity fluctuations display near-wall similarity in local scaling. The present results suggest that turbulence models and wall-modelled large-eddy simulations (Bose \& Park 2018) do not have to take into account curvature effects on turbulence in the near-wall region up to quite strong curvatures and that changes in the wall shear stresses are driven by changes in the turbulence dynamics further away from the walls. 


\section{G. Brethouwer}

Figure 5(a,b) also includes local scaling profiles of streamwise and wall-normal fluctuations in rotating channel flow DNS at $R e=20000$ and $R o=0.15$ on the cyclonic and anticyclonic channel sides. Although rotation and curvature effects differ in the near-wall regions (note that the mean streamline turning rate varies as $U / r$ and vanishes approaching the wall whereas $\Omega$ is constant), the profiles basically collapse, implying that rotation and curvature effects are similar and less significant in the near-wall region at least up to $R o=0.15$. However, stronger rotation with $R o \geq 0.45$ starts to affect near-wall turbulence (Brethouwer 2017), indicating that also stronger curvatures could start to influence near-wall turbulence.

Nagata \& Kasagi (2004), however, did not observe near-wall similarity in their DNSs of curved channel flow; the profiles on the convex and concave sides and for different curvatures showed substantial differences in local scaling, although that was possibly a consequence of a low $R e_{\tau}=150$. In the present DNSs, near-wall similarity also holds less well for the correlation coefficient $\rho=|\overline{u v}| /(\overline{u u} \overline{v v})^{1 / 2}$ on the convex side in the strongly curved cases (figure $5 c$ ), which may explain the early departure of the mean velocity profiles in R3 and R4 seen in figure 2(c). The figure further shows that $u$ and $v$ fluctuations are highly correlated in the outer layer of the concave side in R1 to R4, contributing to the high shear stresses on the concave side. This high correlation points to the presence of coherent turbulent structures.

\section{Reynolds-stress budgets}

To obtain further insights into the effect of curvature on the turbulence, we consider in this section the budget terms of the Reynolds-stress equations. Budgets at lower $\operatorname{Re}_{\tau}$ have been studied by Moser \& Moin (1987) for a mildly curved channel. The streamwise and wall-normal components of the non-dimensional Reynold-stress equations for the present case read, respectively (Hunt \& Joubert 1979; Moser \& Moin 1987)

$$
\begin{aligned}
& \frac{\partial \overline{u^{2}}}{\partial t}=-2 \overline{u v} r \frac{\partial U / r}{\partial r}-4 \overline{u v} \frac{U}{r}-2 \frac{\bar{u} \frac{\partial p}{r}}{\partial \theta}-\frac{1}{r}\left(\frac{\partial r \overline{v u^{2}}}{\partial r}+2 \overline{v u^{2}}\right) \\
& +\frac{1}{R e}\left(\frac{1}{r} \frac{\partial}{\partial r} r \frac{\partial \overline{u^{2}}}{\partial r}+2 \frac{\overline{v^{2}}-\overline{u^{2}}}{r^{2}}\right) \\
& \left.-\frac{2}{\operatorname{Re}} \overline{\left(\frac{\partial u}{\partial r}\right)^{2}}+\overline{\left(\frac{\partial u}{\partial z}\right)^{2}}+\frac{1}{r^{2}} \overline{\left(\frac{\partial u}{\partial \theta}+v\right)^{2}}\right), \\
& \frac{\partial \overline{v^{2}}}{\partial t}=0+4 \overline{u v} \frac{U}{r}-2 \overline{v \frac{\partial p}{\partial r}}-\frac{1}{r}\left(\frac{\partial r \overline{v^{3}}}{\partial r}-2 \overline{v u^{2}}\right) \\
& +\frac{1}{\operatorname{Re}}\left(\frac{1}{r} \frac{\partial}{\partial r} r \frac{\partial \overline{v^{2}}}{\partial r}-2 \frac{\overline{v^{2}}-\overline{u^{2}}}{r^{2}}\right) \\
& -\frac{2}{R e}\left(\overline{\left(\frac{\partial v}{\partial r}\right)^{2}}+\overline{\left(\frac{\partial v}{\partial z}\right)^{2}}+\frac{1}{r^{2}} \overline{\left(\frac{\partial v}{\partial \theta}-u\right)^{2}}\right) \text {. }
\end{aligned}
$$

Here, $p$ is the pressure fluctuation. On the right-hand side of these equations, the terms represent shear production, an additional production resulting from curvature, 


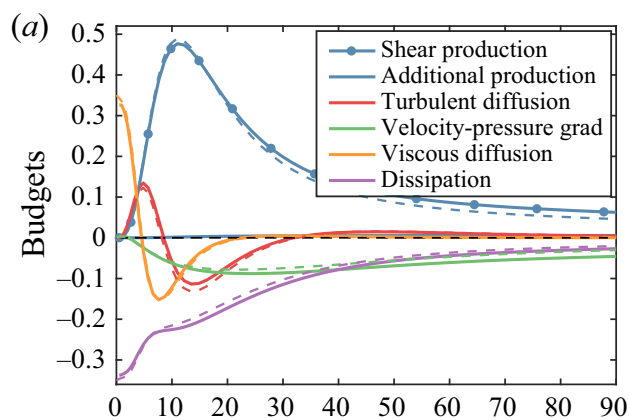

(b)

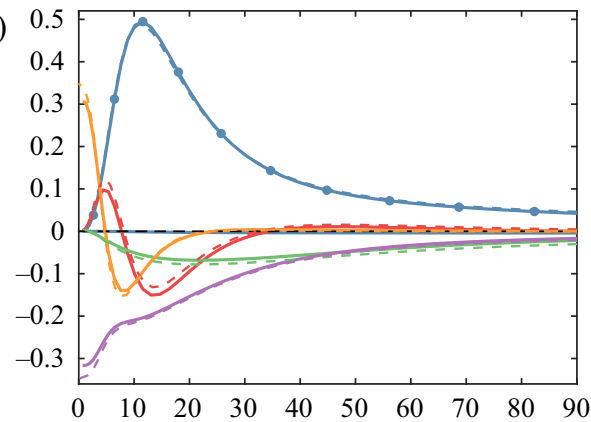

(c)

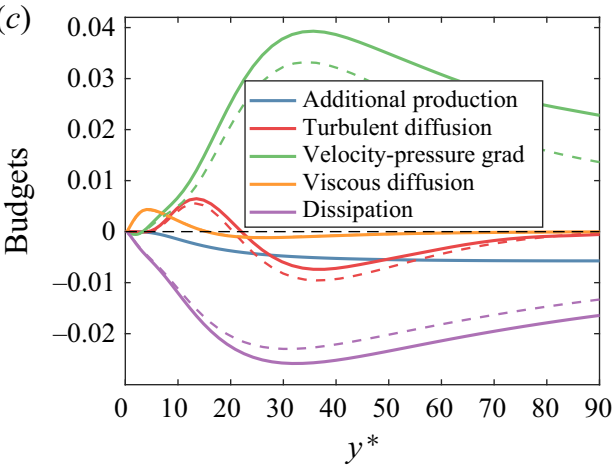

(d)

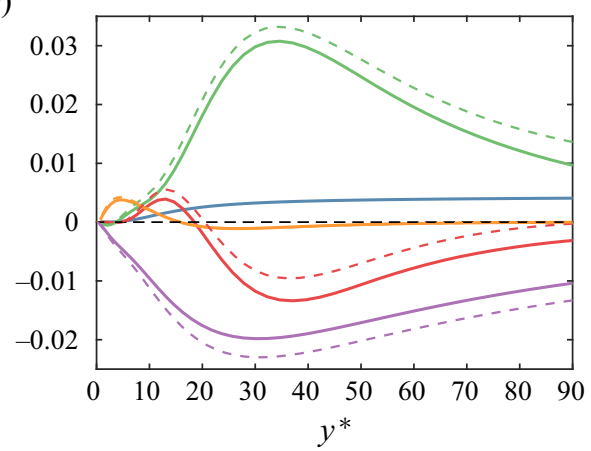

Figure 6. Budgets of the streamwise Reynolds-stress equation (4.1) on the $(a)$ convex and $(b)$ concave sides and budgets of the wall-normal Reynolds-stress equation (4.2) on the (c) convex and $(d)$ concave sides in local wall units. Solid lines: case R3, dashed lines: case R0.

velocity-pressure gradient, turbulent diffusion, viscous diffusion and dissipation, respectively. The shear production term in the equation for $\overline{v v}$ is naturally zero.

Figure 6 shows the budgets of (4.1) and (4.2) in local wall unit scaling near the convex and concave walls in R3. The budgets in R0 are also shown for comparison to elucidate the curvature effect. Only small differences exist in the budgets of $\overline{u u}$ in R0 and R3 on the convex and concave sides and the additional production term in R3 is negligible near the walls. The additional production term is, however, noticeable in the balance equation of $\overline{v v}$, as seen in figure $6(c, d)$. On the concave side it is positive and produces wall-normal Reynolds stresses while on the convex side it reduces them owing to the different sign of $\overline{u v}$. Concave curvature leads to a moderate reduction of the velocity-pressure gradient and dissipation term and augmentation of turbulent diffusion of $\overline{v v}$ while convex curvature has the opposite effect. Similar curvature effects are seen in the other runs and not shown here. To summarize, curvature effects on the Reynolds-stress budgets are small to quite moderate in the near-wall regions.

Figure 7 shows the budgets of (4.1) and (4.2) in R0, R3 and R4 in global wall unit scaling with $u_{\tau}$. The focus is here on the core region, where curvature effects are most prominent. Viscous diffusion is not shown since it only contributes in the viscous sublayer and buffer layer, see figure 6 . The additional production term $\pm 4 \overline{u v} U / r$ resulting from curvature is significant in R3 and R4 and to a lesser extent in R1 and R2 (not shown here) and basically offsets the shear production of $\overline{u u}$ on the concave side until $y \approx-0.3$ to -0.4. The underlying reason is the development of an irrotational mean flow region in curved channel flow DNSs, which implies that the total production, i.e. the sum of shear 


\section{G. Brethouwer}

and additional productions, of $\overline{u u}$ becomes zero and the shear production contribution is basically transferred to the $\overline{v v}$-stress component, as discussed before, see figure $7(d, f)$. The velocity-pressure gradient term in the balance equation of $\overline{u u}$ is negative in R0 but becomes positive in the core region for strong curvatures and contributes to streamwise stresses. In the balance equation of $\overline{v v}$ this term is positive or nearly zero around the centre in $\mathrm{R} 0$ but becomes negative in the core region in R3 and R4. In addition, the magnitude of turbulent diffusion of $\overline{u u}$ and $\overline{v v}$ in the core region grows with curvature. This growth is presumably caused by coherent large-scale motions, which are studied in the next section. Turbulent diffusive transport of kinetic energy by large-scale motions presumably contributes to the strong turbulence in the core region even when, as in R1 and R2, mean shear (figure $2 a$ ) and accordingly shear production are small. It can be further noticed that the additional production term owing to curvature is negative for $\overline{v v}$ and positive for $\overline{u u}$ on the convex side in R3 and R4 and, consequently, transfers energy from wall-normal to streamwise stresses.

\section{Large-scale structures}

Large-scale structures develop in curved channel flows but their characteristics and importance for different curvatures remain elusive. In this section, we therefore study turbulence structures and specifically the large ones as well as their contribution to the Reynolds stresses.

Figure 8 shows visualizations of instantaneous flow fields in planes at a constant radius, i.e. at a constant wall distance. Flow field visualizations of case R0 and rotating channel flow are added for comparison. On the convex side no large-scale structures are visible in R4 (figure 8a) and the other curved channel cases (not shown here). In R4 the curvature is strong enough to cause local flow relaminarization near the convex wall since laminar-like patches without small-scale turbulence develop (figure $8 a$ ), similar to rotating channel flow where the flow relaminarizes on the cyclonic side at sufficiently high rotation rates. This indicates that curvatures as strong as in $\mathrm{R} 4$ and stronger start to affect the near-wall dynamics on the convex side at this $R e$. No large-scale structures are seen in the wall-normal flow field in the outer layer of plane channel flow case R0 (figure $8 b$ ), whereas in R2 (figure 8c) and R1 (not shown here) streamwise streaks are visible with some of them spanning the whole domain in the outer layer of the concave side. The streaks indicate the presence of pairs of large counter-rotating longitudinal vortices, transporting fluid away from the wall, in the mildly curved cases. In R4, streamwise streaks are visible as well but these are less coherent and do not span the whole domain (figure $8 d$ ), suggesting that the large-scale structures are less coherent for strong curvatures. A visualization of the outer layer wall-normal flow field on the anticyclonic side of plane rotating turbulent channel flow at $R e=20000$ and $R o=0.15$ (figure 8e, data from Brethouwer (2017)) shows similar streaks caused by large longitudinal vortices as in the curved channel flow cases.

Figures 9 and 10 show premultiplied two-dimensional energy spectra $k_{x} k_{z} \Phi_{v v}\left(k_{x}, k_{z}\right)$ of the wall-normal fluctuations and $u v$-co-spectra $k_{x} k_{z} \Phi_{u v}\left(k_{x}, k_{z}\right)$, respectively, on the concave side at $y=0.8$ as functions of the streamwise and spanwise wavelengths $\lambda_{x}$ and $\lambda_{z}$ normalized by $\delta$. Here, $k_{x}$ and $k_{z}$ are the streamwise and spanwise wavenumbers, respectively. The energy spectrum $k_{x} k_{z} \Phi_{v v}\left(k_{x}, k_{z}\right)$ in $\mathrm{R} 1$ is similar to that for a plane channel (del Álamo et al. 2006) with a spectral maximum at smaller scales, but also showing contributions from larger scales (figure $9 a$ ). For increasing curvature, the spectral energy of the smaller scales declines whereas that of long and wide scales with $\lambda_{z} \approx 1.5$ grows. The spectral peak also moves from small towards large scales and even very 


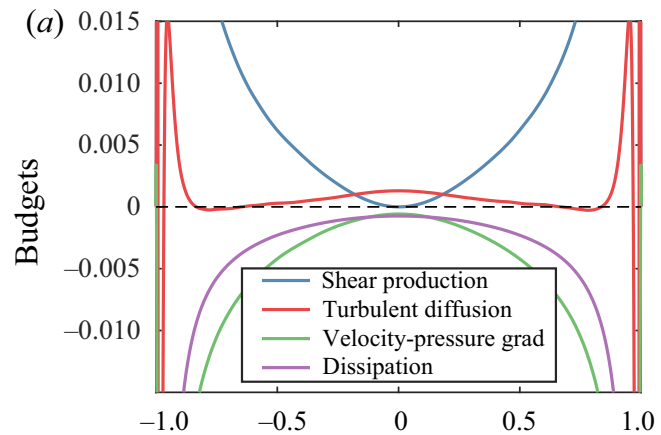

(b)
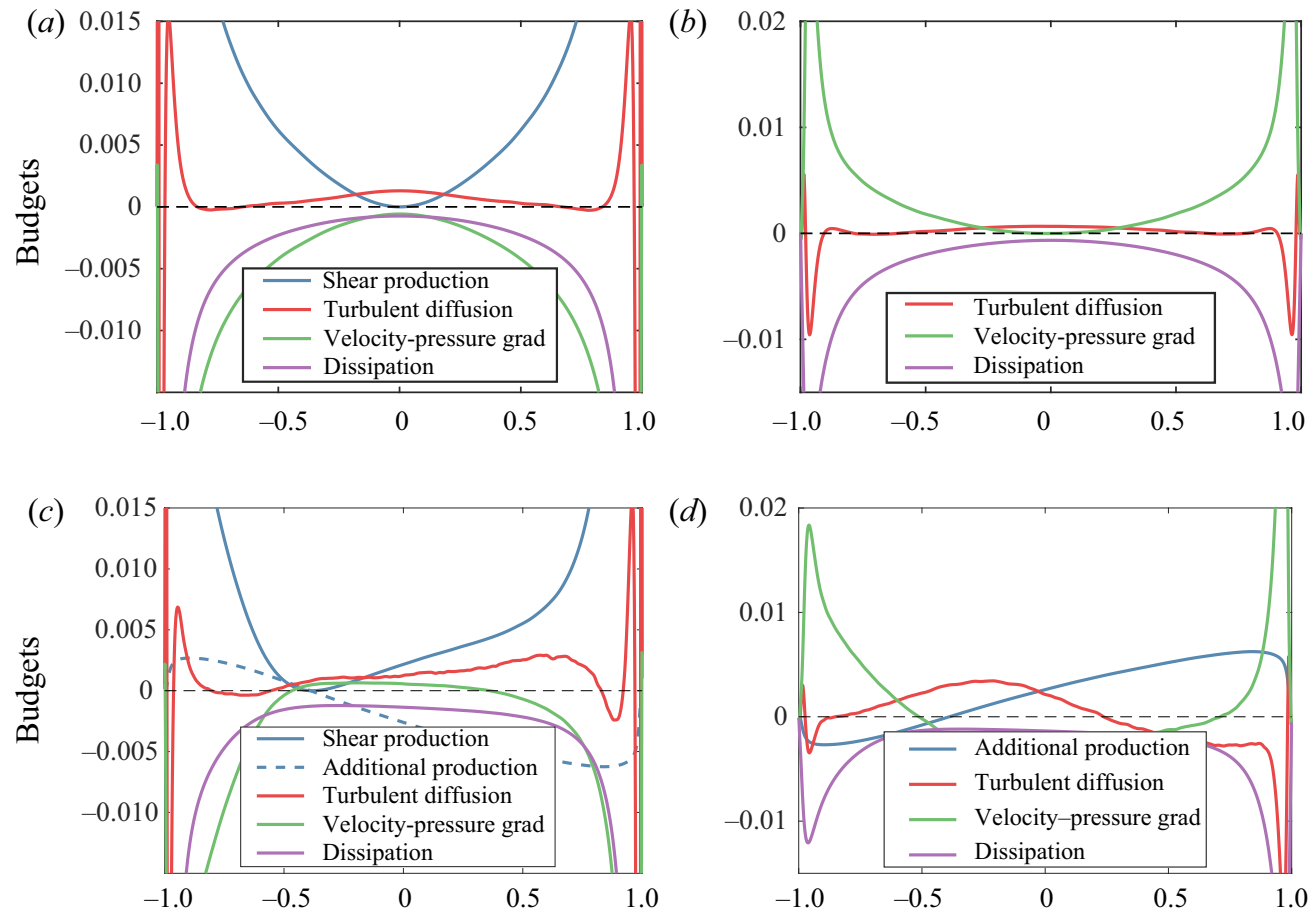

(d)
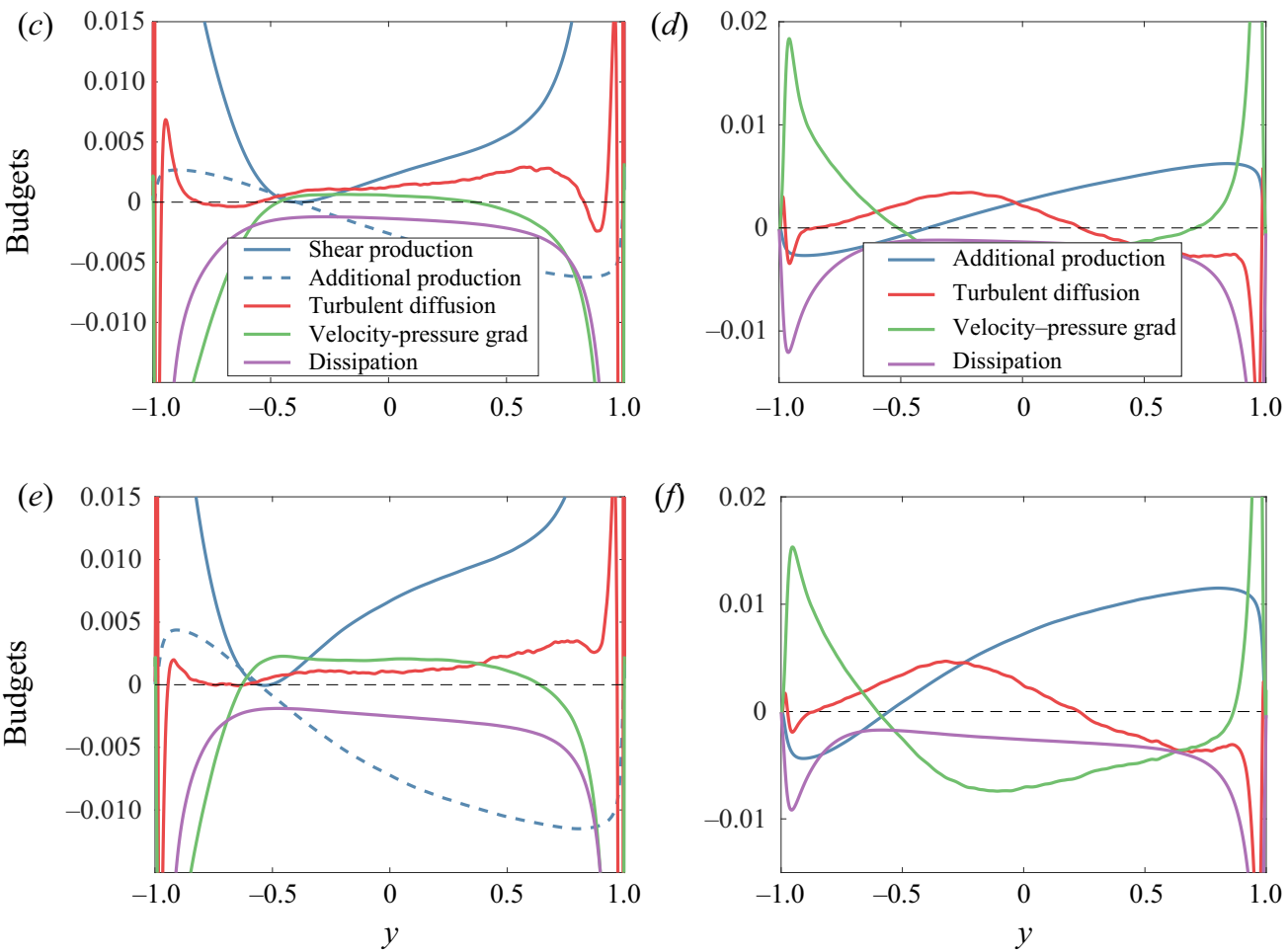

Figure 7. Budgets of the $(a, c, e)$ streamwise Reynolds-stress equation $(4.1)$ and $(b, d, f)$ wall-normal Reynolds-stress equation (4.2) in global wall units; $(a, b) \mathrm{R} 0,(c, d) \mathrm{R} 3$ and $(e, f) \mathrm{R} 4$.

long scales with $\lambda_{x}>10$ have significant energy in R3 and R4, confirming that concave curvature leads to a substantial amplification of larger scales.

Energy spectra measured by Barlow \& Johnston (1988) in the outer layer of a concave boundary layer with $r_{c} / \delta \approx 15$ (with $\delta$ the boundary layer thickness) also show enhanced energy of low frequency, long modes compared with flat boundary layer spectra. The typical length of the large-scale motions was estimated by Barlow \& Johnston (1988) as $3 \delta$ to $5 \delta$. Spectra in R3 and R4, however, show significant energy in modes with $\lambda_{x}>10$, indicating that structures observed in the current DNSs are longer than in Barlow \& Johnston (1988). This could be the result of more homogeneous inflow in the experiments. Longitudinal vortices develop in concave wall boundary layers if the inflow contains 


\section{G. Brethouwer}

(a)
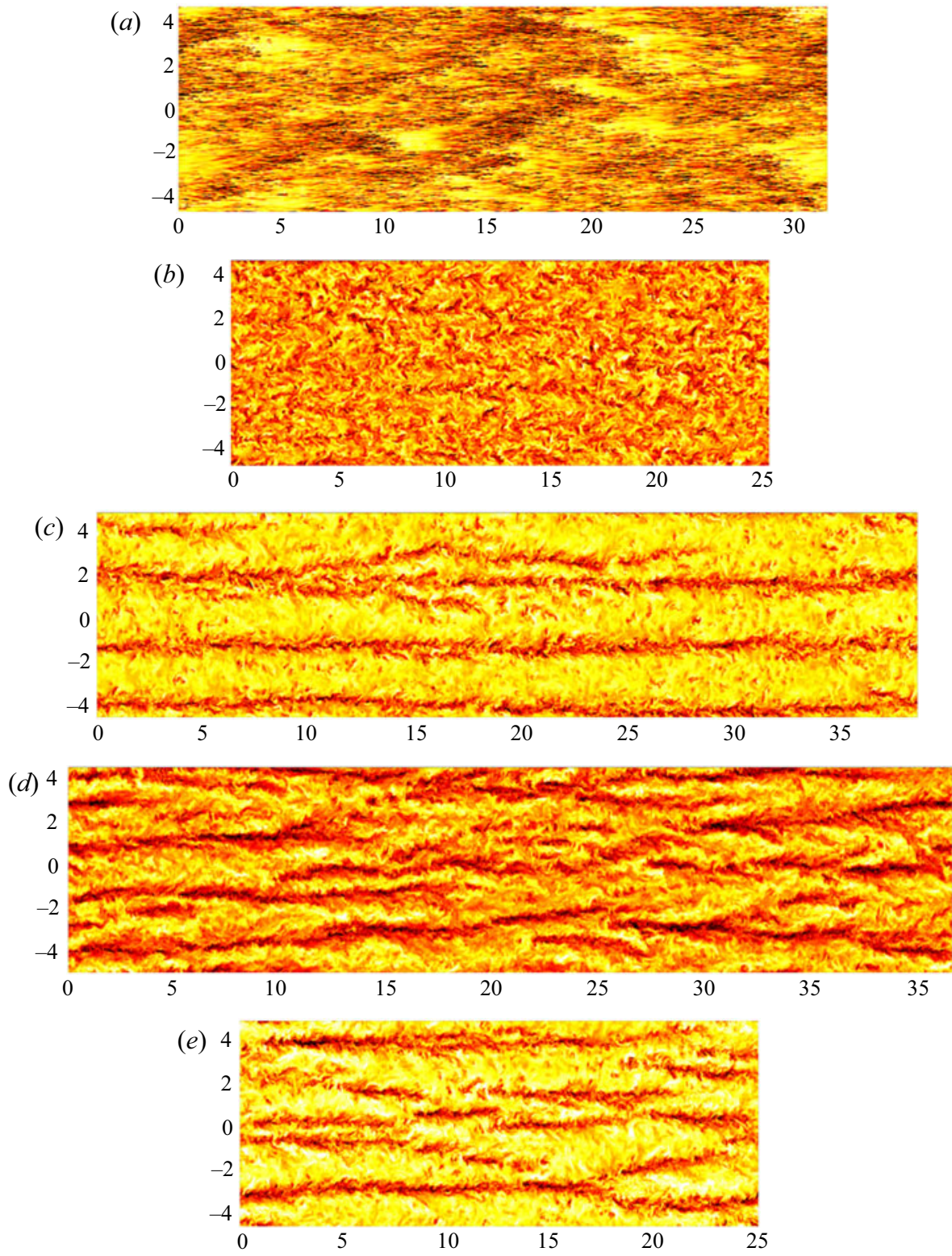

Figure 8. Visualizations of $(a)$ the instantaneous streamwise velocity field near the inner convex wall at $y^{*}=4$ in R4 (red: high velocity), and instantaneous wall-normal velocity field on the concave side at $y=0.6$ in $(b)$ $\mathrm{R} 0,(c) \mathrm{R} 2,(d) \mathrm{R} 4$ and $(e)$ anticyclonic side at $y=0.6$ of rotating channel flow at $R e=20000$ and $R o=$ 0.15 (Brethouwer 2017) (red: negative wall-normal/radial velocity). The horizontal and vertical axes are the streamwise and spanwise directions, respectively.

coherent structures whereas large-scale turbulence structures are enhanced but do not develop into clearly identifiable longitudinal vortices if the inflow lacks coherency (Barlow \& Johnston 1988; Lund \& Moin 1996). In the DNSs the periodic boundary conditions can result in augmented longitudinal vortices (Moser \& Moin 1987).

However, in DNS of a developing flow in a curved channel by Matsubara \& Miura (2017) longitudinal vortices develop as well, even though the turbulent plane channel 
Turbulent flow in curved channels
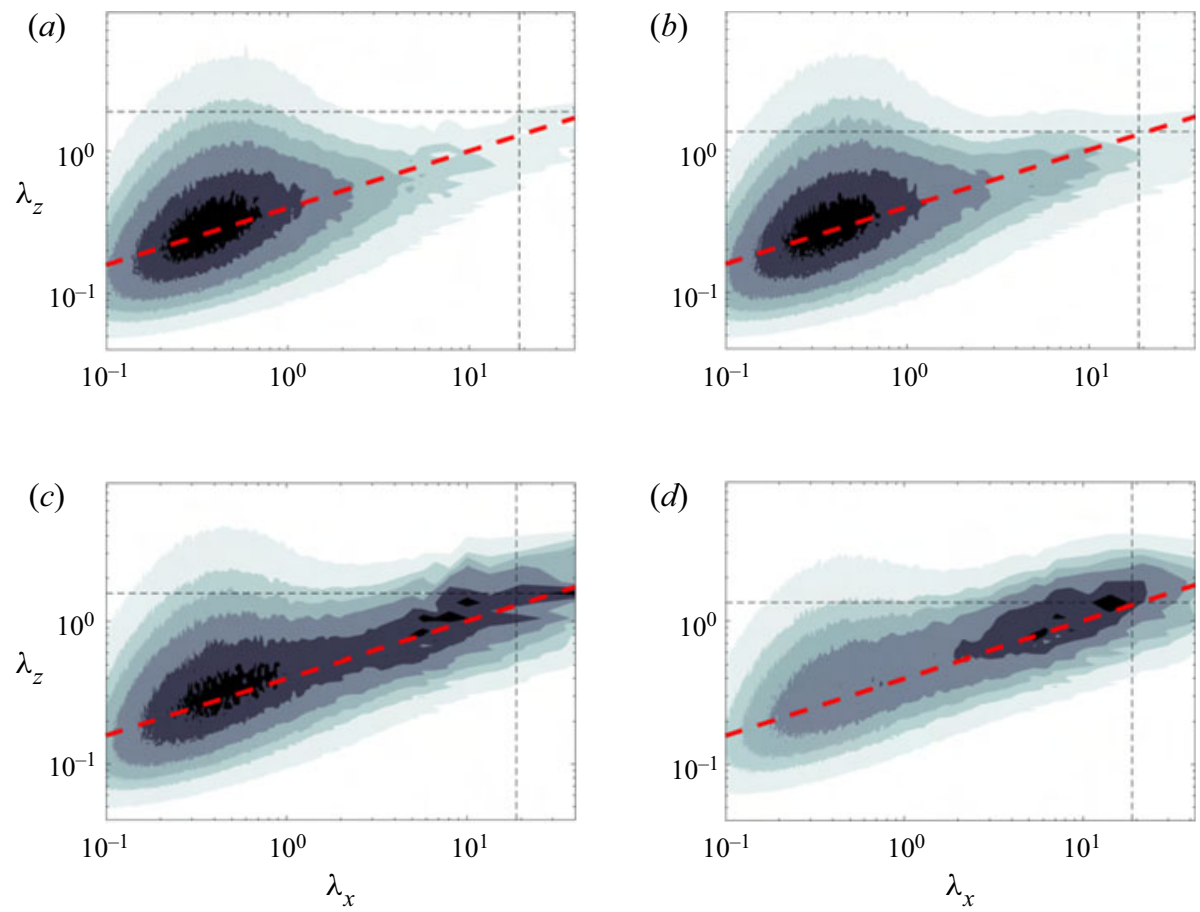

Figure 9. Value of $k_{x} k_{z} \Phi_{v v}\left(k_{x}, k_{z}\right)$ at $y=0.8$ in $(a) \mathrm{R} 1,(b) \mathrm{R} 2,(c) \mathrm{R} 3$ and $(d) \mathrm{R} 4$. Vertical line at $\lambda_{x}=$ $6 \pi$. Horizontal line at (a) $\lambda_{z}=3 \pi / 5$, (b) $\lambda_{z}=3 \pi / 7$, (c) $\lambda_{z}=3 \pi / 6$, (d) $\lambda_{z}=3 \pi / 7$. Red dashed line: $\lambda_{z}=$ $\left(0.1 \lambda_{x}\right)^{0.4}$. Contour levels at $0.8,0.6,0.4,0.28,0.2,0.1$, respectively, times the maximum value.

inflow lacks clear coherency. The vortices in their DNS have a wavelength $\lambda_{z}=2.4$ according to spanwise wall-normal velocity spectra, somewhat wider than in the DNSs here, where the spectral maximum in R3 and R4 suggests a wavelength $\lambda_{z} \approx 1.5$. Also, in DNS of weakly curved channel flow by Moser \& Moin (1987) the longitudinal vortices are considerably larger with a spanwise wavelength of $4 \pi / 3$ than in the present DNSs. This difference could be caused by a low $R e_{\tau}=168$ and constraints resulting from a quite small computational domain in Moser \& Moin (1987). In the current study, we see that large-scale modes are present but these are seemingly less coherent, possibly owing to a higher $R e_{\tau}$, stronger curvature and larger computational domain. Augmented large-scale modes are also obvious in spanwise spectra of the unstable side of spanwise rotating channel flow at $R o=0.15$ (Brethouwer 2017). The large-scale modes become less coherent and smaller at higher $R o$, demonstrating that they reach a maximum size for a certain Ro. In the present study, the large modes do not appear to grow in size for stronger curvatures than in R3 since they are not larger in R4 according to the spectra.

The energetic spectral ridge in figure 9 follows for all curvatures approximately $\lambda_{z} \approx$ $\left(0.1 \lambda_{x}\right)^{0.4}$, indicated by the red dashed line, meaning that turbulence structures become longer yet not much wider with size. Structures with self-similar behaviour, i.e. $\lambda_{z} \sim \lambda_{x}$, are not evident in the spectra, but self-similarity is not expected of TG-like vortices. We should further note that streamwise velocity spectra of turbulent plane wall flows at similar $R e_{\tau}$ are also approximately bounded by $\lambda_{z} \sim \lambda_{x}^{1 / 2}$ (del Álamo et al. 2006; Hwang, Lee \& Sung 2020) and self-similar spectral scaling becomes apparent only at higher $\operatorname{Re}_{\tau}$ (Chandran et al. 2017). Deshpande et al. (2020) demonstrate that self-similar 


\section{G. Brethouwer}
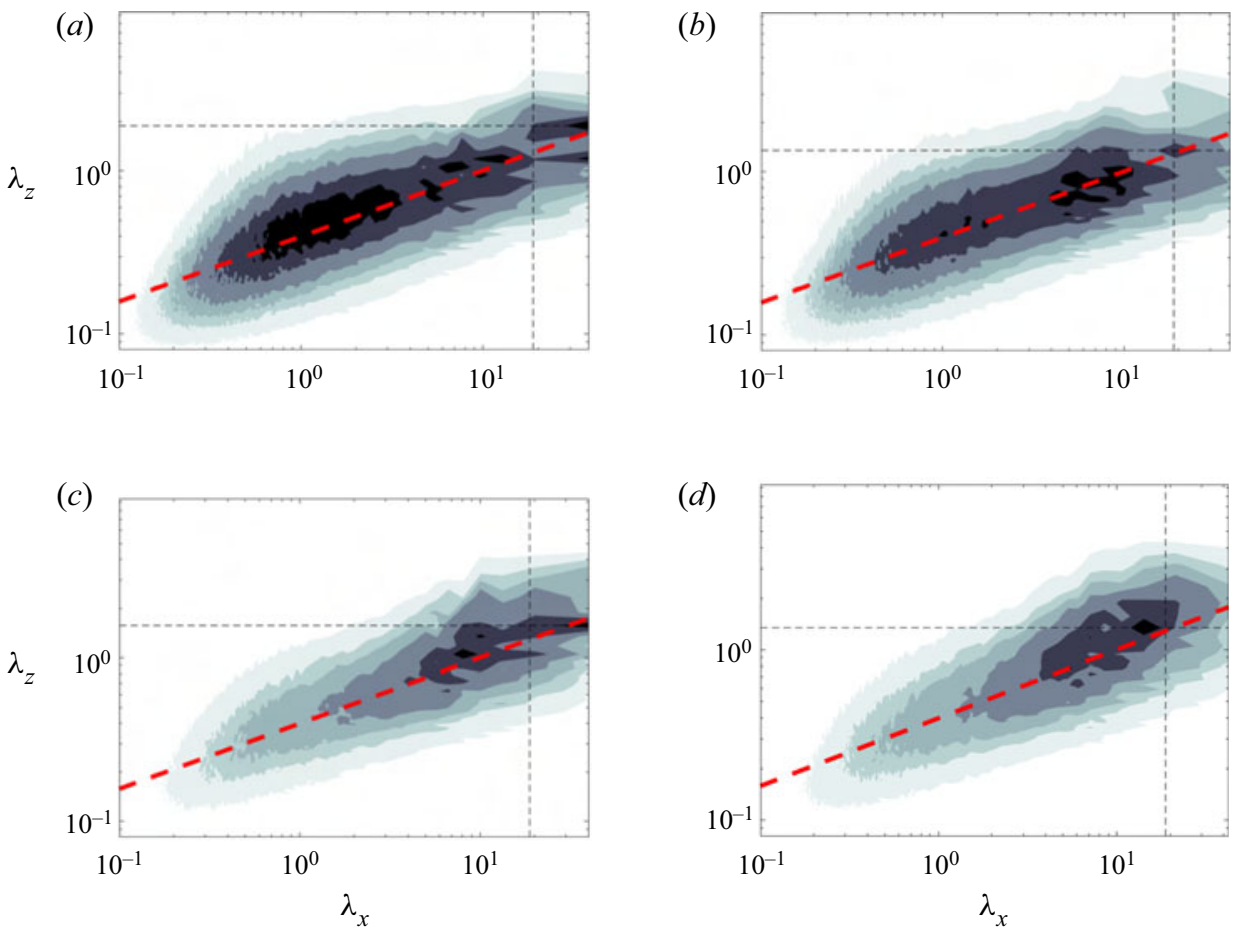

Figure 10. Value of $k_{x} k_{z} \Phi_{u v}\left(k_{x}, k_{z}\right)$ at $y=0.8$ in $(a) \mathrm{R} 1,(b) \mathrm{R} 2,(c) \mathrm{R} 3$ and (d) R4. Vertical line at $\lambda_{x}=6 \pi$. Horizontal line is at (a) $\lambda_{z}=3 \pi / 5$, (b) $\lambda_{z}=3 \pi / 7$, (c) $\lambda_{z}=3 \pi / 6$, (d) $\lambda_{z}=3 \pi / 7$. Red dashed line: $\lambda_{z}=$ $\left(0.1 \lambda_{x}\right)^{0.4}$. Contour levels at $0.8,0.6,0.4,0.28,0.2,0.1$, respectively, times the maximum value.

scaling $\lambda_{z} \sim \lambda_{x}$ of wall attached eddies is obscured by the $\lambda_{z} \sim \lambda_{x}^{1 / 2}$ scaling caused by wall detached eddies at lower $R e_{\tau}$. This suggests that self-similar near-wall structures in curved channel flows, if these exist, only become apparent at higher $R e_{\tau}$.

The co-spectrum $k_{x} k_{z} \Phi_{u v}\left(k_{x}, k_{z}\right)$ of $\mathrm{R} 1$ shows that smaller scales with $\lambda_{x} \sim 1$ and $\lambda_{z} \sim 1 / 3$ as well as very long and wide scales with $\lambda_{z} \sim 1.5$ contribute to momentum transport at $y=0.8$ on the concave side (figure 10a). The spectral ridge again follows approximately $\lambda_{z} \sim \lambda_{x}^{0.4}$, as in a plane channel (del Álamo et al. 2004). With stronger curvature, the spectral peak at smaller scales disappears and a significant part of the momentum transport in the outer layer of the concave side is owing to wide and very long scales (figure $10 c, d$ ). These energetic long and wide scales on the concave side observed in the co-spectra in figure 10 and also before in the energy spectra in figure 9 in the curved channel cases are naturally related to large-scale structures seen in the flow field visualizations in figure $8(c, d)$.

Figure 11 shows premultiplied two-dimensional $u v$-co-spectra $k_{x} k_{z} \Phi_{u v}\left(k_{x}, k_{z}\right)$ at $y^{*}=$ 100 on the convex and concave side in R2 and R4. Wavelengths are again normalized by $\delta$. The co-spectra show that, in both runs, smaller-scale but also large-scale motions contribute to momentum transport on the concave side at this position quite close to the wall. This is especially obvious in R4 with energetic long and wide scales. On the convex side momentum, on the other hand, active large-scale motions are absent and only the smaller-scale contribution is seen in the co-spectra. The turbulence structure is thus obviously different at $y^{*}=100$ on the convex and concave sides, although the velocity fluctuations in local wall units are quite similar, especially in R2 (figure 4). 
Turbulent flow in curved channels
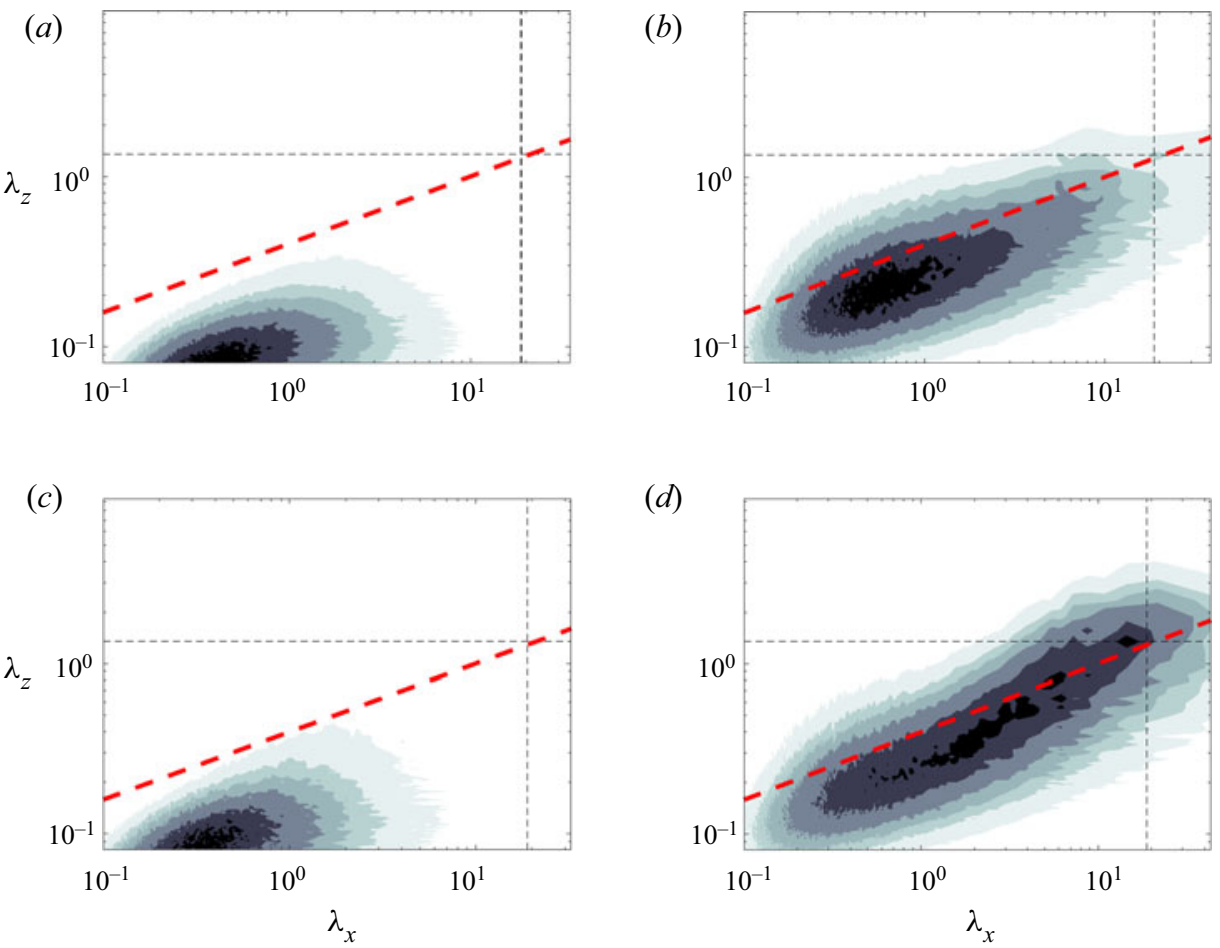

Figure 11. Value of $k_{x} k_{z} \Phi_{u v}\left(k_{x}, k_{z}\right)$ at $y^{*}=100$ in $(a, b) \mathrm{R} 2$ and $(c, d) \mathrm{R} 4$ on the $(a, c)$ convex and $(b, d)$ concave sides. Vertical line at $\lambda_{x}=6 \pi$ and horizontal line at $\lambda_{z}=3 \pi / 7$. Red dashed line: $\lambda_{z}=\left(0.1 \lambda_{x}\right)^{0.4}$. Contour levels at $0.8,0.6,0.4,0.28,0.2,0.1$, respectively, times the maximum value.

The DNS of curved channel flow by Moser \& Moin (1987) could capture one pair of counter-rotating large-scale vortices. Since these were rather coherent, steady and spanned the streamwise domain, Moser and Moin were able to decompose the velocity field into a part owing to the large-scale vortices and a remaining turbulent part by assuming that the vortices are streamwise invariant and not drifting in the spanwise direction. They found that the vortices spanned across the channel and significantly contributed to shear stresses on the concave side but almost negligibly on the convex side. In the present DNSs, we cannot assume that the vortices are quasi-steady and span the whole streamwise domain, as indicated by the visualizations and spectra. Instead of decomposing the velocity field, we therefore use a sharp spectral cutoff filter to study the influence of large-scale modes.

Figure $12(a, b)$ shows the root-mean-square profiles of wall-normal velocity fluctuations and profiles of the mean turbulent shear stress $\overline{u v}$, respectively, of the unfiltered and filtered velocity fields. In the filtered velocity fields only the large-scale modes with wavelengths $\lambda_{x} \geq L_{x} / 4 \simeq 3 \pi \delta$ and $\lambda_{z} \geq L_{z} / 9=\pi \delta / 3$ are retained. In all cases these large-scale modes account for a major part of the wall-normal fluctuations for $-0.1 \lesssim y \lesssim 0.6$ on the concave side. Their energy grows with curvature whereas, on the convex side $(y \lesssim-0.5)$, their contribution is small. The large-scale modes also contribute significantly to momentum transport on the concave side, that is, more than half of the total turbulent momentum transport in R1 to R3 and a third in R4 for $-0.2<y<0.6$ is owing to these large modes. Their contribution on the convex side is again small, demonstrating that convex wall curvature suppresses large-scale motions. Note that in $\mathrm{R} 4$ there is a small region $(-0.56<y<-0.3)$ where the turbulent shear stress $\overline{u v}$ due the large modes has the 


\section{G. Brethouwer}
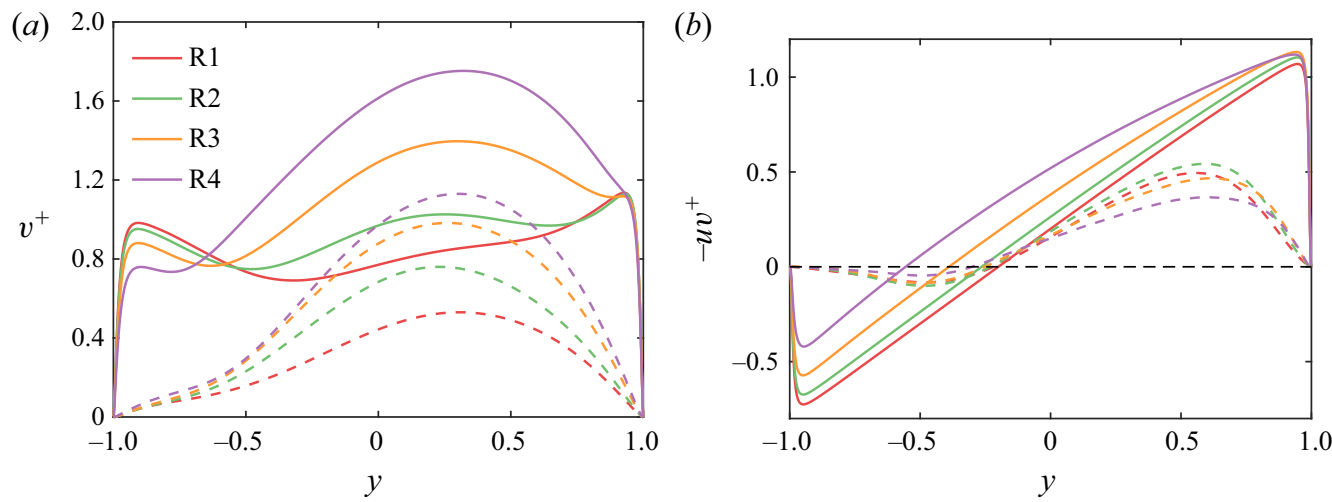

Figure 12. (a) Root-mean-square profiles of wall-normal velocity fluctuations and $(b)$ profiles of the mean turbulent shear stress $\overline{u v}$ in global scaling. The solid lines show the unfiltered results and the dashed lines the contribution of the large-scale motions.

opposite sign to the total turbulent shear stress and therefore negatively contributes. This difference is perhaps caused by significant non-local effects in case of the large-scale modes whereas the smaller scales are instead more influenced and determined by local (shear) effects.

\section{Concluding remarks}

In this study, we have examined the effects of mild to strong longitudinal wall curvature on the fully developed turbulent flow and turbulence structures in a channel by DNS. The $R e_{\tau} \approx 1000$ is high enough to have a clear separation between the near-wall and outer regions.

A substantial difference between the mean wall shear stress and turbulence on the convex and concave walls, respectively, is already observed when $r_{c} / \delta=60$. With increasing curvature, the mean wall shear stress on the concave wall does not change much but on the convex wall it monotonically declines. A log-law region is absent and instead for $r_{c} / \delta \leq 30$ a core region develops, shifted towards the concave channel side, with a nearly constant mean angular momentum, implying neutral stability according to Rayleigh's criterion for centrifugal instability. The effect of streamline curvature is then to redistribute energy from streamwise towards wall-normal velocity fluctuations which are strongly amplified in the outer region of the concave channel side. These observations bear some expected similarities to those in spanwise rotating channel flows. In that case, the mean flow approaches a zero-absolute-mean-vorticity state, also implying neutral stability, and energy is redistributed as well from streamwise towards wall-normal Reynolds stresses on the strongly turbulent anticyclonic channel side (Brethouwer 2017).

Streamline curvature effects are expected to be relatively weak near the walls in curved channels. This expectation is supported by the observed approximate collapse of the mean velocity and root-mean-square velocity fluctuation profiles when scaled by wall units based on the local friction velocity. However, when $r_{c} / \delta=6$, the curvature is strong enough to cause local flow relaminarization near the convex wall.

Budgets of the streamwise and wall-normal Reynolds-stress equations have been computed. These show that, near the walls, streamline curvature has a small influence on the budgets of the streamwise stresses and somewhat larger on these of the wall-normal stresses when scaled with local wall units. In the channel core region, streamline curvature 


\section{Turbulent flow in curved channels}

has naturally a significant effect on the production terms as well as on velocity-pressure gradient and turbulent diffusion terms.

In spanwise rotating channel flow, large-scale longitudinal structures are observed on the anticyclonic side (Brethouwer 2017). Also here there is a similarity to curved channel flow since visualizations show that in my DNSs large, longitudinal vortices are present on the concave side. That observation is further confirmed by premultiplied two-dimensional energy spectra of the wall-normal velocity and co-spectra. These show substantial energy in long and wide modes in the outer region of the concave channel side. However, the longitudinal vortices seem to be less wide and less coherent than in DNS at lower $R e_{\tau}$ with a smaller computational domain (Moser \& Moin 1987), in particular when curvature is strong. Co-spectra at $y^{*}=100$ show that on the convex channel side large-scale active eddies are much less energetic than on the concave side. By applying a low-pass filter, we show that the large-scale modes make an important contribution to the strongly amplified wall-normal fluctuations and high shear stresses on the concave channel side.

Acknowledgements. PRACE is acknowledged for awarding access to Juwels at the Jülich Supercomputing Centre (JSC), Germany, under Prace project 2018194649. SNIC is acknowledged for providing additional computational resources in Sweden. The author is grateful to B.J. Boersma for providing and helping with the DNS code.

Funding. The author further acknowledges financial support from the Swedish Research Council (grant number 621-2016-03533).

Declaration of interests. The author reports no conflict of interest.

Author ORCIDs.

(D) Geert Brethouwer https://orcid.org/0000-0002-9819-2906.

\section{Appendix A. Validation}

Several tests have been carried out to validate the DNS code. Figure 13 shows that the laminar velocity profile in a curved channel obtained from DNS with a wall-normal resolution of $N_{r}=94$ for $r_{i}=1.25$ and 4 matches the analytical solution (Drazin \& Reid 1981) and DNS with a wall-normal resolution of $N_{r}=94$.

For further validation, we have carried out a DNS of fully developed turbulent flow in a curved channel with the same curvature $\left(r_{c}=79\right)$ and the same domain size as the DNS by Moser \& Moin (1987). The resolution is $128 \times 62 \times 128$ in the streamwise, wall-normal and spanwise directions, respectively. Here, $R e=2600$, which resulted in $R e_{\tau}=168$ and $R e_{\tau}^{i}=154$ and $R e_{\tau}^{o}=180$, which are the Reynolds numbers based on the inner and outer friction velocities, respectively. Figures $14(a)$ and 14(b) show the mean velocity profiles and the root-mean-square (r.m.s.) of the velocity fluctuations of the present DNS together with the data from Moser \& Moin (1987). The agreement is in general good. Figure 14(c) shows the turbulent and total shear stress in wall units and the total shear stress according to relation (2.2).

Next, we carried out DNS of fully developed turbulent flow in a channel with $r_{c} \rightarrow \infty$. The domain size is $6 \pi \times 2 \times 2 \pi$ and the resolution is $768 \times 158 \times 512$ in the streamwise, wall-normal and spanwise direction, respectively. Here, $R e=10000$, resulting in $R e_{\tau}=$ 545. Figures $15(a)$ and $15(b)$ show the mean velocity profiles and the r.m.s. of the velocity fluctuations of the present DNS together with the data from del Álamo \& Jiménez (2003) of plane channel flow at $R e_{\tau}=543$. The agreement is very good. Figure 14(c) shows the turbulent shear stress in the present DNS and the DNS by del Álamo \& Jiménez (2003) and the expected and computed total shear stress in wall units. 


\section{G. Brethouwer}

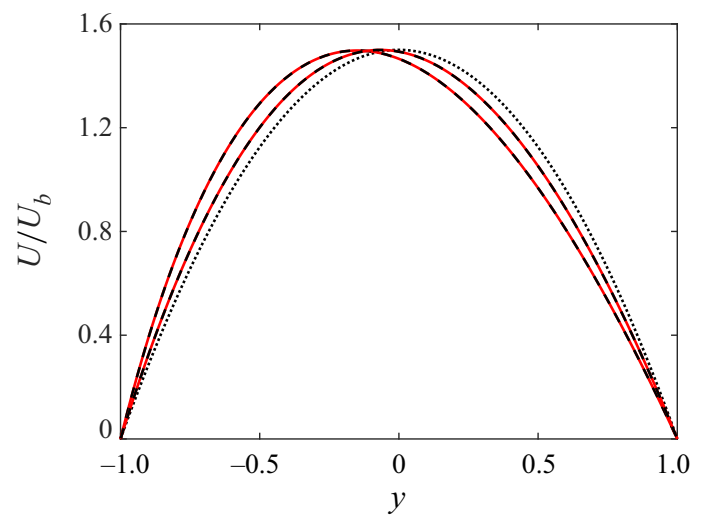

Figure 13. Profiles of $U / U_{b}$ in laminar curved channel flow with $r_{i}=1.25$ and 4; (red solid line) analytical solution (Drazin \& Reid 1981), (dashed line) DNS, (dotted line) laminar Poiseuille flow.
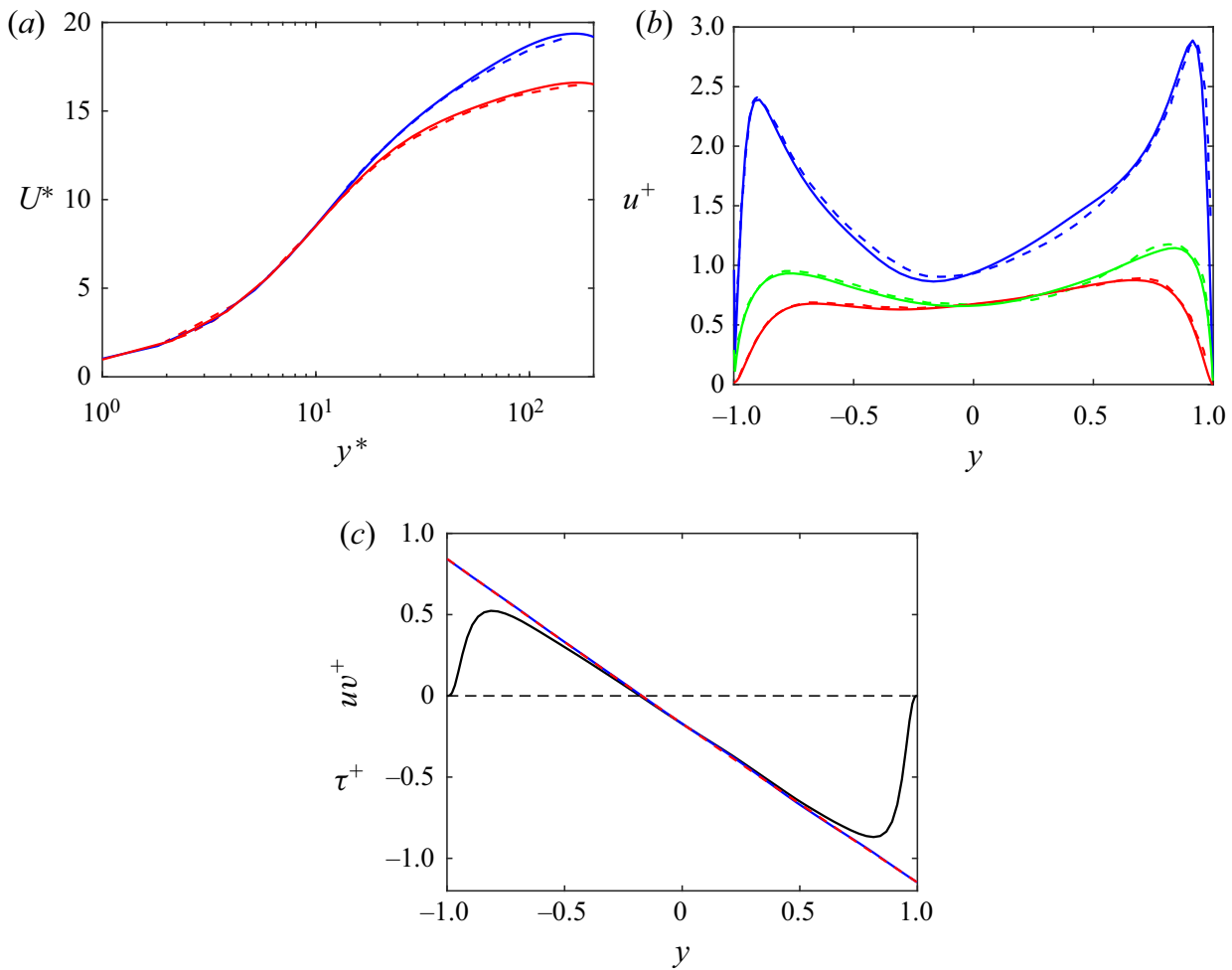

Figure 14. (a) Mean velocity profile in local wall coordinates near the concave (red lines) and convex wall (blue lines). (b) The r.m.s. of the streamwise (blue lines), wall-normal (red lines) and spanwise velocity fluctuations (green lines) in wall units. Dashed lines in $(a)$ and $(b)$ are results of Moser \& Moin (1987) and solid lines are the present results. (c) Values of $-\overline{u v}$ (black line) and total stress (straight blue line) in wall units and total stress according to relation (2.2) (red dashed line). 

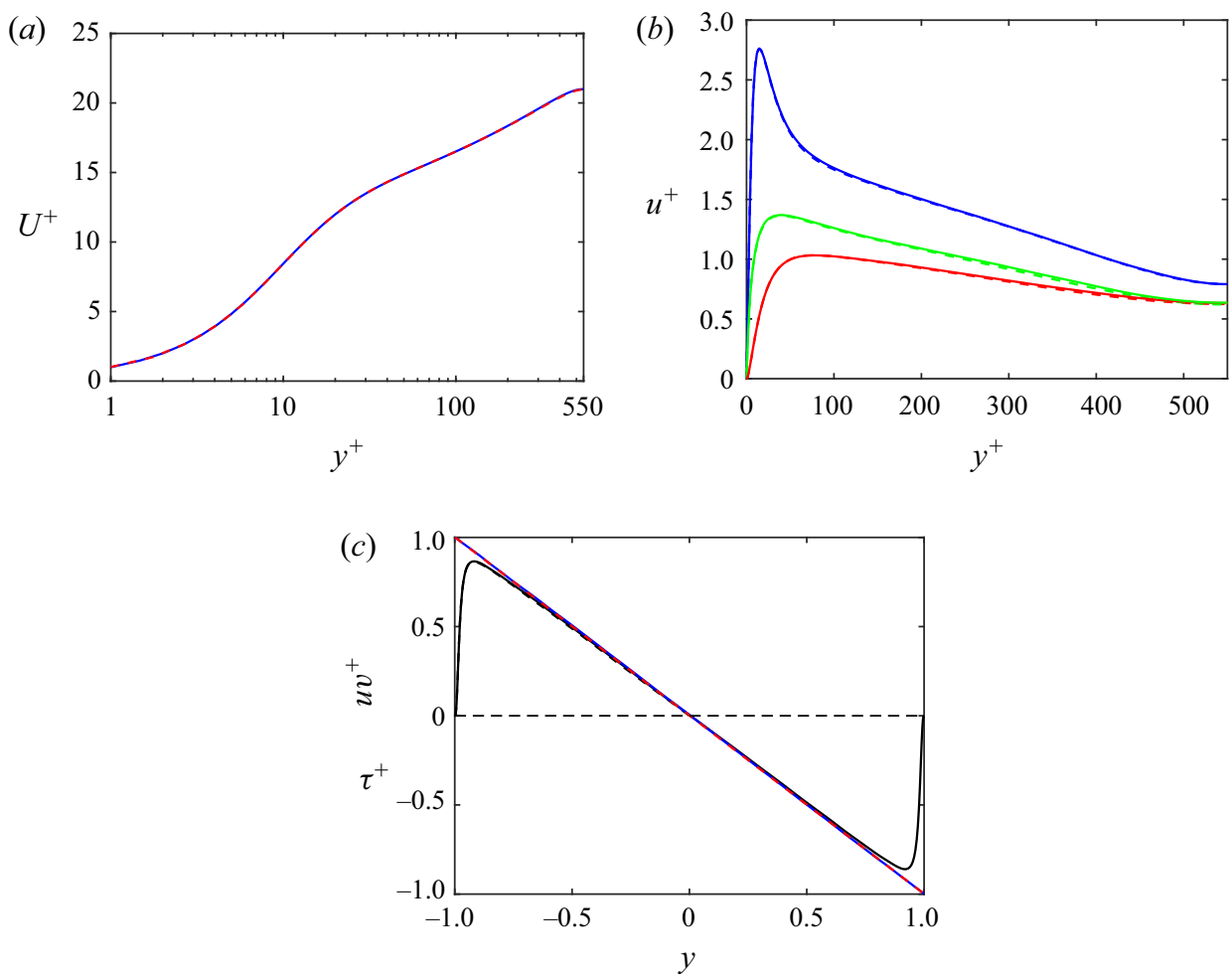

Figure 15. (a) Mean velocity profile in wall coordinates in the DNS by del Álamo \& Jiménez (2003) (blue solid line) and the present DNS (red dashed line). (b) The r.m.s. of the streamwise (blue lines), wall-normal (red lines) and spanwise velocity fluctuations (green lines) in wall units. The dashed lines in $(a)$ and $(b)$ are the results of del Álamo \& Jiménez (2003) and the solid lines are the present results. (c) Values of $-\overline{u v}$ (black solid line present DNS and dashed line data by del Álamo \& Jiménez (2003)) and the total stress (straight blue line) in wall units and the expected total stress (red dashed line).

\section{Appendix B. Momentum balance}

In case of fully developed curved channel flow the governing equation for the streamwise velocity reduces to

$$
\frac{1}{r^{2}} \frac{\partial r^{2} \overline{u v}}{\partial r}=-\frac{1}{\rho r} \frac{\mathrm{d} P}{\mathrm{~d} \theta}+v \frac{\partial}{\partial r}\left(\frac{1}{r} \frac{\partial r U}{\partial r}\right)
$$

since $\partial P / \partial \theta$ is independent of $r$. After multiplication of all terms with $r^{2}$ and integrating (by parts) in the radial direction from $r_{i}$ to $r$ we obtain

$$
\begin{aligned}
r^{2} \overline{u v} & =-\frac{1}{2 \rho} \frac{\mathrm{d} P}{\mathrm{~d} \theta}\left(r^{2}-r_{i}^{2}\right)+v \int_{r_{i}}^{r} r^{2} \frac{\partial}{\partial r}\left(\frac{1}{r} \frac{\partial r U}{\partial r}\right) \mathrm{d} r \\
& =-\frac{1}{2 \rho} \frac{\mathrm{d} P}{\mathrm{~d} \theta}\left(r^{2}-r_{i}^{2}\right)+v\left[r^{2}\left(\frac{\partial U}{\partial r}+\frac{U}{r}\right)\right]_{r_{i}}^{r}-2 v \int_{r_{i}}^{r} \frac{\partial r U}{\partial r} \mathrm{~d} r \\
& =-\frac{1}{2 \rho} \frac{\mathrm{d} P}{\mathrm{~d} \theta}\left(r^{2}-r_{i}^{2}\right)+v\left[r^{2}\left(\frac{\partial U}{\partial r}+\frac{U}{r}\right)-\left.r_{i}^{2} \frac{\partial U}{\partial r}\right|_{r_{i}}-2 r U\right]
\end{aligned}
$$




\section{G. Brethouwer}

From that follows

$$
-\frac{1}{\rho} \frac{\mathrm{d} P}{\mathrm{~d} \theta}=\frac{2 v}{r_{o}^{2}-r_{i}^{2}}\left(\left.r_{i}^{2} \frac{\partial U}{\partial r}\right|_{r_{i}}-\left.r_{o}^{2} \frac{\partial U}{\partial r}\right|_{r_{o}}\right)=\frac{1}{2 r_{c} \delta}\left(r_{i}^{2} u_{\tau i}^{2}+r_{o}^{2} u_{\tau o}^{2}\right),
$$

where $u_{\tau i}^{2}=\left.v(\partial U / \partial r)\right|_{r_{i}}$ and $u_{\tau o}^{2}=-\left.v(\partial U / \partial r)\right|_{r_{o}}$. The mean pressure gradient at the centreline is $-(\mathrm{d} P / \mathrm{d} \theta) /\left(\rho r_{c}\right)$ where $r_{c}=\left(r_{i}+r_{o}\right) / 2$. In a similar spirit as for plane channel flow we define the friction velocity then as

$$
u_{\tau}^{2}=-\frac{1}{2} \frac{r_{o}-r_{i}}{\rho r_{c}} \frac{\mathrm{d} P}{\mathrm{~d} \theta}=\frac{1}{2 r_{c}^{2}}\left(r_{i}^{2} u_{\tau i}^{2}+r_{o}^{2} u_{\tau o}^{2}\right) .
$$

Eliminating the pressure in (B2) with the help of (B3) and (B4) and some rewriting gives finally for the momentum balance across the channel

$$
\tau=v\left(\frac{\partial U}{\partial r}-\frac{U}{r}\right)-\overline{u v}=\frac{1}{4 r^{2} r_{c} \delta}\left[r_{i}^{2}\left(r_{o}^{2}-r^{2}\right) u_{\tau i}^{2}+r_{o}^{2}\left(r_{i}^{2}-r^{2}\right) u_{\tau o}^{2}\right] .
$$

\section{REFERENCES}

DEl Álamo, J.C.\& Jiménez, J. 2003 Spectra of the very large anisotropic scales in turbulent channels. Phys. Fluids 15, L41-L44.

Del Álamo, J.C., Jiménez, J., Zandonade, P. \& Moser, R.D. 2004 Scaling of the energy spectra of turbulent channels. J. Fluid Mech. 500, 135-144.

Del Álamo, J.C., Jiménez, J., Zandonade, P. \& Moser, R.D. 2006 Self-similar vortex clusters in the turbulent logarithmic region. J. Fluid Mech. 561, 329-358.

BARLOW, R.S. \& JOHNSTON, J.P. 1988 Structure of a turbulent boundary layer on a concave surface. J. Fluid Mech. 191, 137-176.

Boersma, B.J. 2011 A 6th-order staggered compact finite difference method for the incompressible Navier-Stokes and scalar equations. J. Comput. Phys. 230, 4940-4954.

Bose, S.T. \& PARK, G.I. 2018 Wall-modeled large-eddy simulation for complex turbulent flows. Annu. Rev. Fluid Mech. 50, 535-561.

BRADSHAW, P. 1969 The analogy between streamline curvature and buoyancy in turbulent shear flow. J. Fluid Mech. 36, 177-191.

BRAdshaw, P. 1973 The effects of streamline curvature on turbulent flow. Tech. Rep. AGARD-AG-169. Advisory Group for Aerospace Research and Development Paris (France).

Brauckmann, H.J., Salewski, M. \& Eckhardt, B. 2016 Momentum transport in Taylor-Couette flow with vanishing curvature. J. Fluid Mech. 790, 419-452.

Brethouwer, G. 2017 Statistics and structure of spanwise rotating turbulent channel flow at moderate Reynolds numbers. J. Fluid Mech. 828, 424-458.

Chandran, D., Baidya, R., Monty, J.P. \& Marusic, I. 2017 Two-dimensional energy spectra in high-Reynolds-number turbulent boundary layers. J. Fluid Mech. 826, R1.

Deshpande, R., Chandran, D., Monty, J.P. \& Marusic, I. 2020 Two-dimensional cross-spectrum of the streamwise velocity in turbulent boundary layers. J. Fluid Mech. 890, R2.

DRAZIN, P.G. \& REID, W.H. 1981 Hydrodynamic Stability. Cambridge University Press.

Durbin, P.A. 2018 Some recent developments in turbulence closure modeling. Annu. Rev. Fluid Mech. 50, 77-103.

ESKINAZI, S. \& YEH, H. 1956 An investigation on fully developed turbulent flows in a curved channel. J. Aero. Sci. 23, 23.

GiLlis, J.C. \& Johnston, J.P. 1983 Turbulent boundary-layer flow and structure on a convex wall and its redevelopment on a flat wall. J. Fluid Mech. 135, 123-153.

Hoffmann, P.H., Muck, K.C. \& BRADShaw, P. 1985 The effect of concave surface curvature on turbulent boundary layers. J. Fluid Mech. 161, 371-403.

Hunt, I.A. \& Joubert, P.N. 1979 Effects of small streamline curvature on turbulent duct flow. J. Fluid Mech. 91, 633-659.

HwAng, J., LEE, J.H. \& SUNG, H.J. 2020 Statistical behaviour of self-similar structures in canonical wall turbulence. J. Fluid Mech. 905, A6. 


\section{Turbulent flow in curved channels}

Lee, M. \& Moser, R.D. 2015 Direct numerical simulation of turbulent channel flow up to $R e_{\tau} \approx 5200$. J. Fluid Mech. 774, 395-415.

Lund, T.S. \& Moin, P. 1996 Large-eddy simulation of a concave wall boundary layer. Intl J. Heat Fluid Flow 17, 290-295.

MATSUbARA, K. \& MiURA, T. 2017 Spatially advancing turbulent flow and heat transfer in a curved channel at friction-velocity-based Reynolds number 550. Intl J. Heat Mass Transfer 108, 2433-2443.

Matsubara, K. \& Muromoto, T. 2019 Two-point correlation and integral scale of spatially advancing curved channel flow at friction-velocity-based Reynolds number 550. Intl J. Heat Fluid Flow 77, 31-39.

Moser, R.D. \& Moin, P. 1987 The effects of curvature in wall-bounded turbulent flows. J. Fluid Mech. $175,479-510$.

Muck, K.C., Hoffmann, P.H. \& BRAdshaw, P. 1985 The effect of convex surface curvature on turbulent boundary layers. J. Fluid Mech. 161, 347-369.

NAGATA, M. \& KASAGI, N. 2004 Spatio-temporal evolution of coherent vortices in wall turbulence with streamline curvature. J. Turbulence 5, 017 .

Patel, V.C. \& Sotiropoulos, F. 1997 Longitudinal curvature effects in turbulent boundary layers. Prog. Aerosp. Sci. 33, 1-70.

Peeters, J.W.R., Pecnik, R., Rohde, M., van der Hagen, T.H.J.J. \& Boersma, B.J. 2016 Turbulence attenuation in simultaneously heated and cooled annular flows at supercritical pressure. J. Fluid Mech. 799, 505-540.

Wattendorf, F.L. 1935 A study of the effect of curvature on fully developed turbulent flow. Proc. R. Soc. Lond. A 148, 565-598. 